\title{
Direct comparison of PDF and scalar dissipation rates between LEM simulations and experiments for turbulent, premixed methane air flames
}

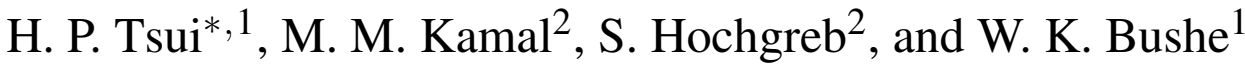 \\ 1 *Department of Mechanical Engineering, University of British Columbia \\ Vancouver, BC, V6T 1Z4, Canada \\ 2 Department of Engineering, University of Cambridge \\ Cambridge, CB2 1PZ, United Kingdom
}

\begin{abstract}
We present a direct comparison between the predicted and measured probability density functions (PDF) of the reaction progress variable and conditioned values of the scalar dissipation rates (SDR) in premixed turbulent flames. The predictions are based on simulations of premixed flames using the linear-eddy model (LEM), parameterised by a wide range of integral length scales and turbulent Reynolds numbers. The experimental results are highly spatially resolved temperature and species data from the CambridgeSandia swirl burner. The LEM simulations display remarkable accuracy in capturing the features observed experimentally. Further, the results reveal that the LEM calculated PDF and SDR for premixed flames remain relatively steady under a variety of turbulent conditions, including variations in the integral length and turbulent Reynolds number. In general, it appears to be practical to use representative pseudo-turbulent PDF and SDR models for a range of turbulence intensities and length scales.
\end{abstract}

\section{Introduction}

Several practical models for turbulent premixed combustion rely on an accurate representation of the probability density function (PDF) of a reaction progress variable, which is often parameterised by the mean and variance of that progress variable [1-6]. These presumed PDF approaches are often implemented in conjunction with tabulated chemical variables to achieve detailed chemistry calculations in turbulent combustion simulations. Such models have been developed for both the Reynolds-averaged Navier-Stokes (RANS) and large eddy simulation (LES) paradigms. Previous work has shown that the accuracy of these methods depends to a considerable extent on the accuracy of the function presumed for the PDF of the progress variable [1].

A number of different presumed PDF models have been previously investigated for premixed combustion. The often used $\beta$-PDF does recover the extreme properties expected of the true PDF, such as $\delta$ functions at the zero and unity extremes of reaction progress for maximal variance, and single $\delta$ functions at the mean for zero variance. However, it fails to reproduce the shape of the true PDF in more general cases [1]. The issue is related to the fact that the shape of the true PDF appears to be a function of how the chemical reaction rates vary as a function of the progress variable; hence, different chemical kinetics lead to different shapes of the PDF of progress variable. The form of the $\beta$-PDF - which is of course entirely independent of the chemical kinetics - can lead to significant inaccuracies. Most critically, there can be a biasing error, as the discrepancies tend to occur at the same values of the progress variable for any particular flame.

One of the primary concerns in the design of modern engines is the reduction of harmful pollutants; specifically, the current generation of numerical models must be able to predict both the thermodynamic properties of the reacting mixture and the formation of these minor species with sufficient accuracy to resolve the parts per million produced. A prominent example is the prediction of prompt flame $\mathrm{NO}_{x}$ via

*corresponding author, hongtsui@alumni.ubc.ca 
the Fenimore pathway [7]. The predicted $\mathrm{NO}_{x}$ values from this mechanism are strongly affected by the choice of the PDF model, as this pathway is sensitive to the predicted temperatures and flame profile in the reactive regions.

In an attempt to account for the effects of chemistry on the shape of the PDF, Bray et al. [8] proposed using a premixed laminar flame to model the functional dependence of the PDF on progress variable. The proposed probability dependence of the flame existing at any given state is inversely proportional to the magnitude of the gradient of the temperature. Their original formulation only provided coverage for flames with very high variance. Jin et al. [1] then proposed a modification to the Bray PDF that extends the original formulation to cover all possible mean and variance combinations. This is accomplished by truncating the PDF shape function as needed to match the mean and variance parameters. It was found to significantly improve the fit of the PDF to that extracted from Direct Numerical Simulation (DNS) results. A shortcoming of this method is that, at the point of truncation, the model PDF has a sharp drop to zero, whilst the true PDF tends to be more rounded.

To address this issue, a one-dimensional turbulent method was proposed to take the place of the typical laminar flame calculation to tabulate pseudo-turbulent PDF models for RANS and LES closures. The Linear-Eddy Model, an inexpensive one dimensional stochastic mixing model, has demonstrated the ability to capture important effects from the interaction between chemistry and turbulence on the PDF distributions sufficiently well [9-11]. This model provides us with a mechanism to investigate the general flame characteristics at very high turbulence intensities, much beyond the capability of current DNS strategies. In turn, it permits us to analyze the behavior of the PDF constructed at these highly turbulent states.

While the LEM has been implemented to investigate the shape of the PDF distributions [9, 10], such simulations were performed to analyze the PDF for specific flames. The current study is primarily interested in the tabulation of a PDF lookup table useful for subsequent RANS and LES flame computations (a pre-processing operation not unlike pre-calculating the $\beta$-function and storing that in a lookup table). At first, there was a suggestion that one ought to try to match the turbulence statistics in the LEM calculations to those that one expects to find in the later turbulent flame calculation. Indeed, a possibility is that one might need to add a dimension to the lookup table (something like the local turbulent Reynolds number) to account for variations in local turbulence properties in the turbulent flame calculation and their effect on the shape of the PDF. This is a large part of the motivation for the LEM work presented here: do the turbulence properties affect the shape of the PDF? If so, how? How important is it to match the LEM turbulence properties used in generating the PDF lookup table to those that will be found in the turbulent flow to be calculated later?

A related question can be asked about the local gradient of progress variable in a premixed turbulent flame which is closely related to the scalar dissipation rate (SDR). For premixed combustion, the SDR, $\chi_{c}$, of a temperature-based reaction progress variable is,

$$
\chi_{c}(T, \phi)=\alpha_{c}(T, \phi) \nabla c \cdot \nabla c,
$$

where $\alpha_{c}(T, \phi)$ and $\nabla c$ are the thermal diffusivity at the local temperature and equivalence ratio and the gradient of the progress variable, respectively.

The SDR is an important quantity to both non-premixed and premixed combustion modeling [12, 13]. It is an unclosed term that appears in the transport equation for the variance of progress variable, where $\chi_{c}$ directly measures the decay rate of fluctuations through turbulent micromixing [13]. Since the burn rate of many combustion processes depends on the contact area and local gradient between the reactants, it is reasonable for most combustion models to assume that the mean burning rate of the flames either explicitly or implicitly depends on the scalar dissipation rate. For example, Conditional Moment Closure (CMC) uses the scalar dissipation rate conditioned by the progress variable to calculate micromixing [13]; not surprisingly, modeling the conditional scalar dissipation term $\chi_{c} \mid c^{*}$ conditioned on the local and global progress of reaction emerges as one of the main difficulties in applying CMC to turbulent premixed flames [14].

The same LEM method can be used to generate a model for the conditional scalar dissipation, where one simply conditionally averages the scalar dissipation in the LEM temperature profiles that are used to 
construct the PDF model. The unconditional mean SDR can be obtained by convolving the conditional SDR with the model PDF. This allows for the construction of pseudo-turbulent PDF and SDR models that are perfectly consistent with one another. While it is possible to obtain PDF and SDR distributions for premixed combustion from DNS, the associated cost is generally prohibitive for flows with relevant

turbulent conditions [1]. More importantly, studies typically tend to focus on the analysis of the PDFs and SDRs at specific points within the domain. This leads to the problem that although DNS and experiments can provide valuable insight to the behavior of the PDF and SDR [15-17], they cannot provide usable input models for subsequent RANS and LES combustion calculations, which is the primary motivation behind the current work. The incorporation of turbulence characteristics in the PDF distributions could provide a solution to the deficiencies seen in a number of current PDF models, such as the ad hoc $\beta$-pdf or the laminar approaches. Having more accurate PDF and SDR models would be beneficial to a number of RANS and LES strategies as closures for turbulent premixed combustion typically rely on some variant of the PDF or SDR model for the reaction progress variable as the input [12,13].

Recent detailed measurements of species and temperature have been made by the Cambridge-Sandia swirl burner [16, 18-20]. This swirl burner was designed specifically to explore the influence of stratification on the flame. However, the very detailed nature of the scalar and velocity measurements have made the data set attractive as a target for premixed flame model validation as well [21, 22]. In particular, the comprehensive database allows conditioning on a number of different variables, including equivalence ratio (for the stratified flames), temperature (or progress of reaction) or any other suitable scalar.

In this paper we use the experimental dataset to obtain detailed PDFs of the progress of reaction. The temperature is used to characterise the extent of reaction, for direct comparison with the PDFs generated from LEM simulations for three different swirl (and turbulence) levels. We consider the measured and computed variances, as well as the detailed shape of the PDFs in the comparison. In addition, we are also able to directly evaluate the unconditional mean SDR values obtained from experiment and LEM simulations. In the following sections we discuss the numerical approach, followed by a summary of the experiments and the data treatment used.

\section{Numerical Conditions: Premixed Combustion}

\subsection{Linear-Eddy Model}

The Linear-Eddy Model has been demonstrated to replicate the flow statistics for simple turbulent conditions with acceptable accuracy [23-27]. Given the one-dimensional nature of the model, the computational costs remain relatively low for most practical cases. Here, the LEM is used in a pre-processing manner for the tabulation of discrete PDF and SDR models, which can be implemented in subsequent RANS and LES applications.

The LEM can be divided into two modules. The deterministic component consists of the usual onedimensional gas dynamics evolution equations, whereas the stochastic component consists of random eddy events. The turbulence concept of LEM postulates a random process that rearranges fluid elements along a line in order simulate the chaotic vortices that appear in turbulent fields. These one-dimensional vortices, known as triplet maps, generate discontinuous fluid motions, which lead to a random walk of fluid elements. The eddy event frequency per unit length of the domain is governed by [26],

$$
\lambda_{L E M}=\frac{54}{5} \frac{v e_{t}}{C_{\lambda} l_{0}^{3}} \frac{\left(l_{0} / l_{k}\right)^{5 / 3}-1}{1-\left(l_{k} / l_{0}\right)^{4 / 3}},
$$

where $v, R e_{t}, l_{0}$ and $l_{k}$ are the kinematic viscosity, turbulent Reynolds number, the integral length scale and the Kolmogorov scale, respectively. The empirical parameter $C_{\lambda}$ should typically be tuned to the flame in question for LEM studies [10]; however, we are primarily interested in investigating the changes in the PDF models associated with variations in the turbulent fluctuations and integral length scales. As such, $C_{\lambda}$ is held constant for consistency between cases. The value of 15.0 for $C_{\lambda}$ is adopted from [10]. Similarly, the parameter used to scale the Kolmogorov length, $N_{\eta}$, which typically requires tuning to case specific conditions, is also held at a constant value of 1.0 for all of the LEM cases. This parameter is applied to the inertial scaling law in the following form,

$$
l_{k}=N_{\eta} l_{0} R e_{t}^{-3 / 4} .
$$


The deterministic and stochastic modules are implemented simultaneously during the simulation to achieve a pseudo-turbulent effect; this coupling between the stochastic advective process and deterministic evolution equations in a one dimensional computational domain permits the LEM to simulate turbulent flows with higher Reynolds numbers than multi-dimensional models. The current LEM variant follows

the formulation of [28] to accommodate premixed turbulent reacting flows.

To a large extent, the applicability of LEM depends on the homogeneity of the turbulence and the degree of which the problem can be characterised in one dimension within the simulation domain. This suggests that the method may fail where there is significant coupling between turbulence non-homogeneity at the small scales and the flow field. For many problems in premixed combustion, the spatial scales where reactions take place tend to be of the order of the laminar flame thickness, and in many cases, experiments show that at such small scales of turbulence, its characteristics have decayed to conditions that are reasonably isotropic and uniform.

\subsection{LEM Simulation Methods}

A global, six-step mechanism by Chang et al. [29] designed to simulate premixed methane-air combustion is implemented to generate the flame profiles. The six global reaction rates have been reduced by approximately $8 \%$ to achieve the correct unstrained laminar flame propagation speed observed in the reference solution from Cantera's GRI MECH 3.0 [30] calculation. This extra calibration procedure ensures increased accuracy for our value of equivalence ratio of $0.73^{1}$.

All of the species properties are calculated using CHEMKIN-II [31], including specific heats, diffusion coefficients, thermal conductivities and enthalpies. The thermodynamic coefficients are based on the CHEMKIN Thermodynamic Data Base [32]. Figure 1 illustrates the flame solutions from Cantera and the Chang mechanism. Mixture-averaged transport is adapted to reduce computational time. The inflow mixture was set to atmospheric pressure and $294 \mathrm{~K}$ at an equivalence ratio of 0.73 . The calculated laminar flame speed, $S_{L}$, under these conditions is $0.214 \mathrm{~m} / \mathrm{s}$, and the calculated laminar flame thickness, $\delta_{f}$, is $588 \mu \mathrm{m}$. The overall flame thickness, propagation speed, equilibrium temperature and mass fractions of major and minor species are sufficiently well matched between the reduced Chang and full GRI mechanisms.

The LEM simulations are conducted with a mixed first order upwind and second order centered spatial scheme. Explicit time steps are taken in order to cope with the stochastic nature of the model. The instantaneous temperature profiles are recorded at regular time intervals on the order of $1 \%$ of a large eddy turnover time for the freely propagating flames. Some 3,500 to 16,000 temperature profiles are stored for post-simulation construction of the LEM PDF models. The exact number of profiles required to create a statistically converged PDF model is dependent primarily on the turbulence intensity of the flame. Ten prototype turbulent premixed flames with various integral length scales and turbulent Reynolds numbers are tested. The flow parameters are varied from case to case such that the prototype flames adhere to predetermined locations on the Borghi diagram, as illustrated in Figure 2. The LEM parameters used to simulate the freely propagating flames at the prescribed turbulent conditions are summarised in Table 1.

Throughout the paper, we use a temperature-based reaction progress variable:

$$
c=\frac{T-T_{0}}{T_{e}(\phi)-T_{0}},
$$

where $T_{e}(\phi)$ is the equilibrium temperature at the equivalence ratio $\phi$ and $T_{0}$ is the initial temperature. From the simulations or experiments, it is possible to construct a PDF with the following form,

$$
P\left(c^{*} ; x, t\right) \approx P\left(c^{*} ; \bar{c}, \bar{c}^{2}\right),
$$

where $\bar{c}$ and $\bar{c}^{\prime 2}$ are the mean and variance of the progress variable, and $c^{*}$ is the discretized variable representing the continuous $c$ space. In another words, the PDF at any space and time within the simulation

\footnotetext{
${ }^{1}$ Reduced mechanisms are typically designed to operate over a range of thermodynamic conditions. However, due to a more restricted degree of freedom from the reduced number of steps, the level of accuracy may not be identical over the range of operations. For this numerical study, we are primarily interested at premixed flames with $\phi=0.73$; thus, we optimize the reduced mechanism for this particular equivalence ratio.
} 

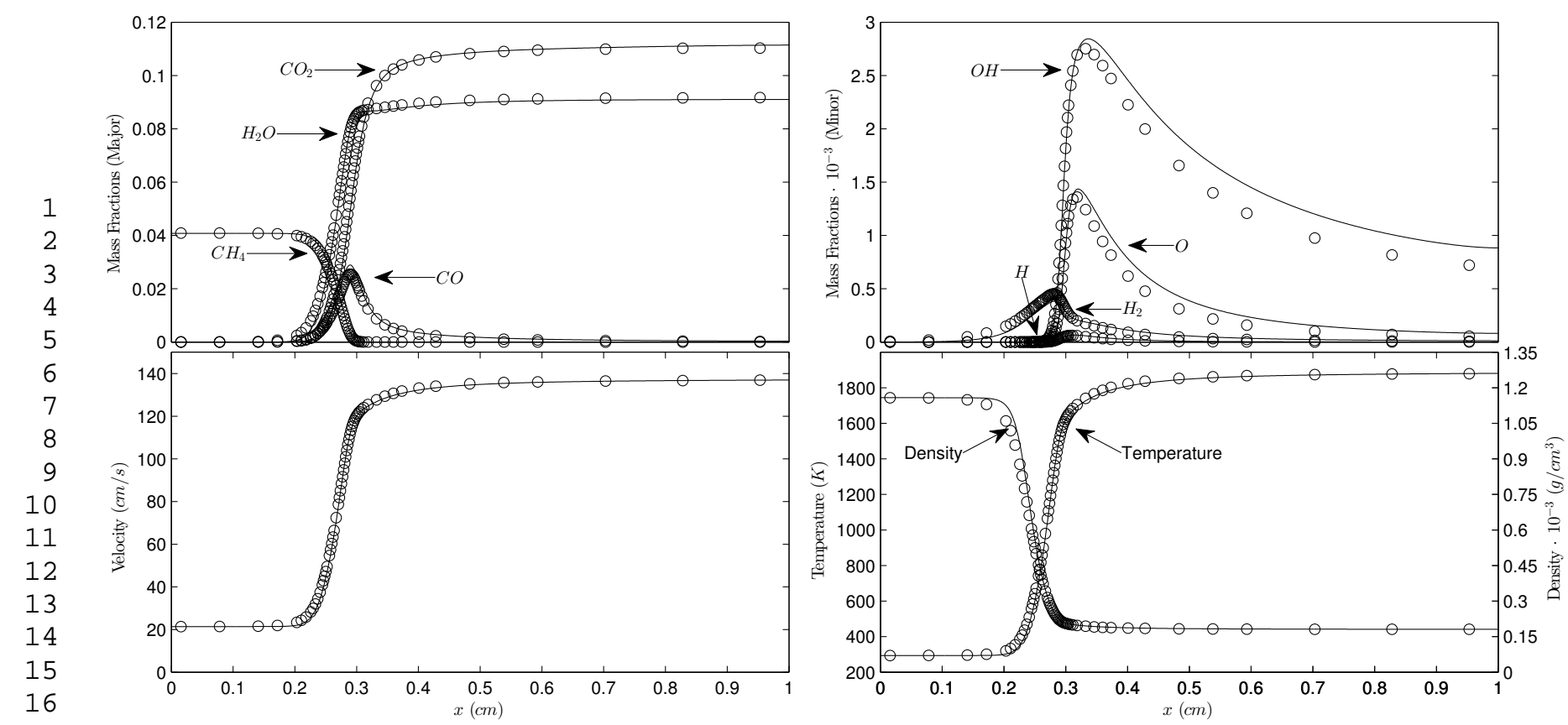

Figure 1. Premixed laminar flame solution at an equivalence ratio of 0.73 for the 6 -step global mechanism (line) and Cantera [33] (symbols).

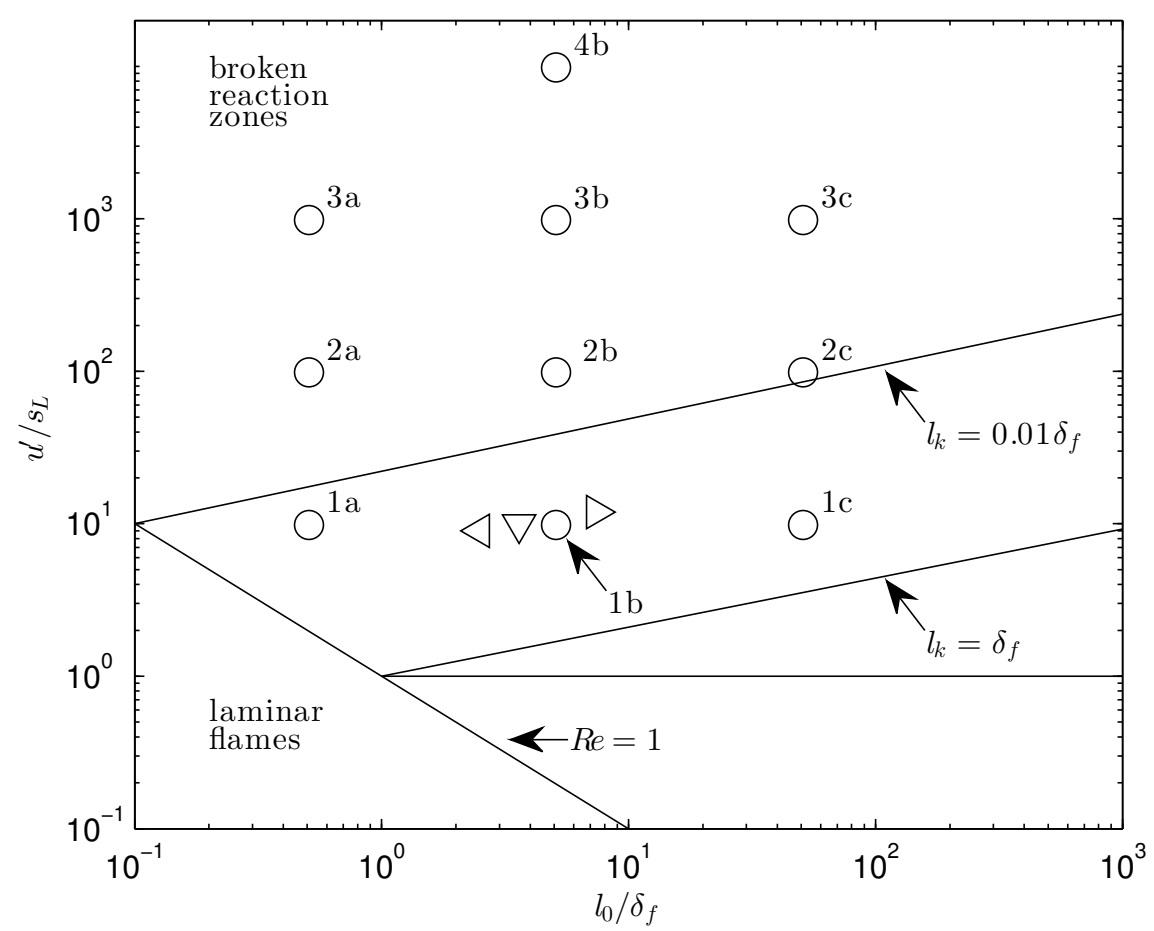

Figure 2. Borghi diagram showing locations of the ten prototype LEM flames (1a to $4 \mathrm{~b}$ ). The LEM test cases are represented by 'o'. The experimental flames are represented by triangles: SwB1 (' $\triangleleft$ '), SwB2 (' $\nabla$ '), and SwB3 (' $\triangleright$ '). It has been found that increasing the integral length beyond an order of magnitude above the laminar flame thickness while holding the turbulent fluctuations constant does not significantly alter the PDF profiles for the LEM simulations.

domain can be approximated by the mean and variance of the distribution. Such a formulation is compatible with several current models for the turbulence and chemistry interactions for turbulent premixed combustion [1-4]. A detailed description of the LEM PDF formulation can be found in [11], while a brief overview of the construction of the LEM PDF is provided in Section 4. Furthermore, we define a normalised variance, $\bar{c}_{n}{ }_{n}^{2}$, determined by the limiting variance of a perfectly segregated mixture of hot and 


\begin{tabular}{|c|c|c|c|c|c|c|c|}
\hline Case & $R e_{t}$ & $l_{0}(\mathrm{~cm})$ & $l_{k}(\mathrm{~cm})$ & $u^{\prime} / S_{L}$ & Domain $(\mathrm{cm})$ & Min. cells/cm & Cells used \\
\hline 1a & 5 & 0.02992 & $8.95 \cdot 10^{-3}$ & 9.826 & 1.0 & 670 & 1,000 \\
\hline 1b & 50 & 0.2992 & $1.59 \cdot 10^{-2}$ & 9.826 & 1.0 & 337 & 1,000 \\
\hline 1c & 500 & 2.992 & $2.83 \cdot 10^{-2}$ & 9.826 & 4.0 & 318 & 1,272 \\
\hline 2a & 50 & 0.02992 & $1.59 \cdot 10^{-3}$ & 98.26 & 1.0 & 3,771 & 3,772 \\
\hline 2b & 500 & 0.2992 & $2.83 \cdot 10^{-3}$ & 98.26 & 1.0 & 2,121 & 2,122 \\
\hline 2c & 5000 & 2.992 & $5.03 \cdot 10^{-3}$ & 98.26 & 4.0 & 1,193 & 4,772 \\
\hline 3a & 500 & 0.02992 & $2.83 \cdot 10^{-4}$ & 982.6 & 1.0 & 21,204 & 21,204 \\
\hline 3b & 5000 & 0.2992 & $5.03 \cdot 10^{-4}$ & 982.6 & 1.0 & 11,924 & 11,924 \\
\hline 3c & 50000 & 2.992 & $8.95 \cdot 10^{-4}$ & 982.6 & 4.0 & 6,706 & 26,824 \\
\hline 4b & 50000 & 0.2992 & $8.95 \cdot 10^{-5}$ & 9826 & 1.0 & 67,053 & 67,054 \\
\hline
\end{tabular}

Table 1. Relevant LEM simulation parameters: $l_{0}$ and $l_{k}$ are the integral and Kolmogorov scales. Other constant parameters are invariant between the cases, including $\delta_{f}, S_{L}, C_{\lambda}$ and $N_{\eta}$, which are respectively, $588 \mu \mathrm{m}, 0.214 \mathrm{~cm} / \mathrm{s}, 15.0$ and 1.0. A minimum of 1,000 computational cells are used for each simulation.

cold gases as:

$$
{\overline{c^{\prime}}}_{n}^{2}=\frac{\bar{c}^{\prime 2}}{\bar{c}(1-\bar{c})}
$$

This formulation is more suitable for the discrete tabulation of the PDF table, as the boundary values conveniently vary between zero and unity.

\section{Experimental Conditions: Stratified Swirl Burner}

The flames and experimental techniques have been described in previous papers [16, 19, 34], so the methods are summarised briefly here. A turbulent flame is stabilised on a bluff body, with reactants fed through two concentric streams at specified inner and outer equivalence ratios $(\phi)$. In the present paper, only premixed cases are considered. Swirl can be added to the flame by splitting the outer stream through a tangential inlet.

The global equivalence ratio for the flame is nominally 0.75 (measured via species is $0.73+/-0.018$ ), and the range of local equivalence ratios spans $0.375-1.125$. Scalar data obtained from Rayleigh/Raman/COLIF line measurements at $103 \mu \mathrm{m}$ resolution allows the behaviour of key combustion species $-\mathrm{CH}_{4}, \mathrm{CO}_{2}$, $\mathrm{CO}, \mathrm{H}_{2}, \mathrm{H}_{2} \mathrm{O}$ and $\mathrm{O}_{2}$ - to be probed within the instantaneous flame front. Simultaneous cross-planar OHPLIF is used to determine the orientation of the instantaneous flame normal in the scalar measurement window, allowing real gradients of the temperature and hence the progress variable to be obtained.

The operating conditions considered in the present analysis are listed in Table 2. Measurements were taken at six heights along the centerline of the flame, by collecting data in $6 \mathrm{~mm}$ linear segments. The notation $\mathrm{SwBN}_{z}$ is used to denote cases at different locations, where $N$ is the case number and $z$ is the axial coordinate. We are considering three premixed flames under different swirl conditions.

\begin{tabular}{|c|c|c|c|}
\hline Case & SFR $^{(1)}$ & $\mathrm{SN}^{(2)}$ & $u^{\prime} / S_{L}^{(3)}$ \\
\hline SwB1 & 0 & 0 & 10.2 \\
\hline SwB2 & 0.25 & 0.26 & 12.2 \\
\hline SwB3 & 0.33 & 0.45 & 14.3 \\
\hline
\end{tabular}

Table 2. Operating Conditions: (1) SFR = ratio of split flow to swirlers to total flow, (2) $\mathrm{SN}=$ measured swirl number, ratio of tangential to axial momentum, (3) Maximum total $u^{\prime} / S_{L}$ at the midpoint of the flame brush at $z=30 \mathrm{~mm}[20,35]$.

The bulk velocity in the outer annulus, $U_{o}=18.7 \mathrm{~m} / \mathrm{s}$, is set at more than twice the value of the velocity in the inner annulus, $U_{i}=8.3 \mathrm{~m} / \mathrm{s}$, so as to generate substantial levels of shear, and thus turbulence, between the two flows. Co-flow air was supplied around the outer annulus with a bulk velocity $U_{c o}=$ 
$0.4 \mathrm{~m} / \mathrm{s}$ to provide well-characterised boundary conditions. The Reynolds numbers derived from the bulk velocities at the exit geometry are $R e_{i}=5,960$ for the inner flow and $R e_{o}=11,500$ for the outer flow.

Multi-scalar laser diagnostics were applied at the Turbulent Combustion Laboratory in Sandia National Laboratories, and extensively described in [16, 19, 34]. The diagnostics setup allows for the line

measurement of temperature (Rayleigh scattering) and major species (Raman scattering and CO-LIF) at $103 \mu \mathrm{m}$ projected pixel resolution with simultaneous cross planar OH-PLIF at $48 \mu \mathrm{m}$ projected pixel resolution. Signal to noise ratios are of the order of 150 for temperatures, and about 60 for equivalence ratio, with estimated accuracies of $2 \%$ and $5 \%$, respectively. As the optical resolution of the RamanRayleigh-LIF measurements is smaller than the spatial sampling rate, the resolution of the temperature and major species measurements is limited by the sampling resolution $(103 \mu \mathrm{m})$ and the laser beam diameter $\left(0.22 \mathrm{~mm}, 1 / e^{2}\right)$. The optical resolution $\left(1 / e^{2}\right)$ of the OH-PLIF measurement is between 98 and $144 \mu \mathrm{m}$ and therefore the spatial resolution of the OH-PLIF measurements is limited by the optical resolution rather than the sampling resolution. The OH-PLIF cross planar technique allows the flame normal in $3 \mathrm{D}$ to be assessed relatively to the measurement line, thus allowing the real gradients to be obtained by projection. Radial profiles were obtained by moving the burner horizontally in $4 \mathrm{~mm}$ steps, producing overlapping steps in the relative position of the $6 \mathrm{~mm}$ wide measurement window, with 300 laser shots taken at each step. Radial profiles were taken at axial increments of $10 \mathrm{~mm}$ above the burner exit to capture changes in flame structure with axial distance. Substantially larger datasets $(5,000-30,000$ shots) were taken at the intersection of the mean flame brush and the mixing layer for stratified flames only. Here we confine ourselves to the premixed cases only, within $2.5 \%$ of the nominal value of 0.75 .

\section{Construction of PDF Models}

\subsection{LEM Flame Profiles}

Figure 3 illustrates a few selected LEM temperature profiles for two prototype flames of varying turbulence intensities. It can been seen that the influence of the triplet maps becomes more pronounced and modify a larger portion of the flame as the turbulent Reynolds number increases. Consequently, the turbulent temperature profiles become more distinguishable from the laminar counterpart, with larger effective flame thicknesses. These perturbations propagate through the flame and reduce in strength until they either blend into the flame profile or get transported out of the domain.

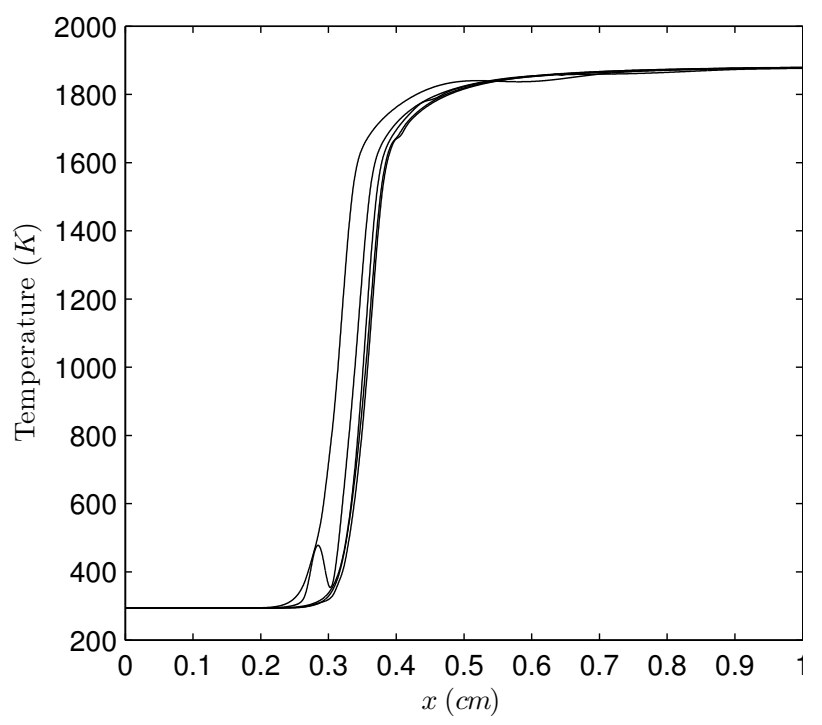

(a) Case $2 \mathrm{~b}\left(R e_{t}=500\right)$.

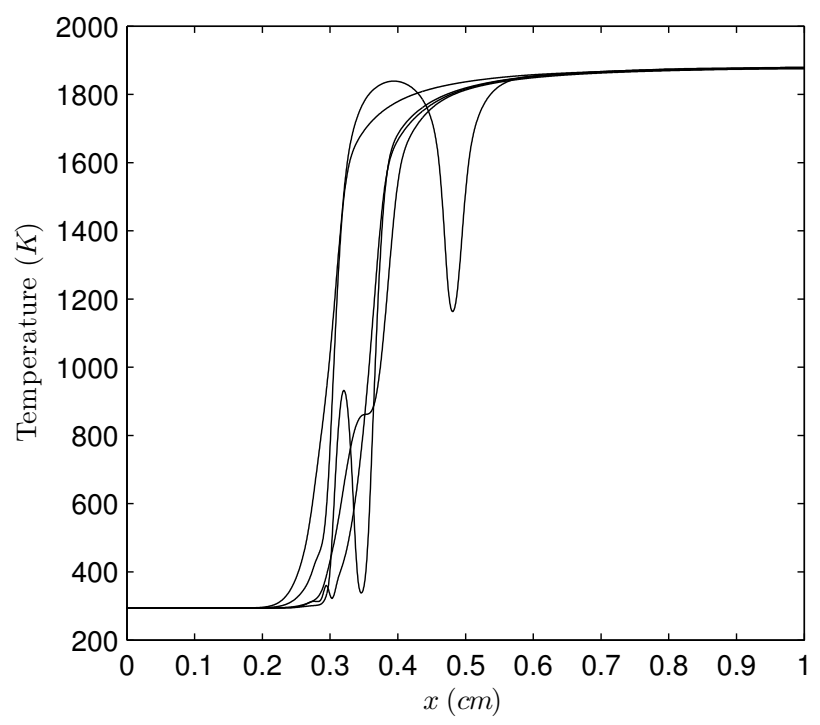

(b) Case $4 \mathrm{~b}\left(R e_{t}=50,000\right)$.

Figure 3. Characteristic LEM temperature profiles of two prototype flames at different $R e_{t}$. The individual profiles on each graph are separated by at least one large eddy turnover time.

At first inspection, it appears that the average temperature gradients should increase with the number of triplet maps implemented per unit time; however, the diffusion mechanism from the evolution equations quickly diminishes the sharp discontinuities introduced in the slope of the temperature field. As a 
consequence, the flame begins to broaden as it recovers from this perturbed state. The net effect is to decrease the overall conditional averages of the gradients when considered across the entire width of the flame. The temperature profiles depicted in Figure 3 can then be transformed into the progress variable space via Equation 4.

\subsection{LEM PDF Construction}

The method used to construct the LEM PDF models is first discussed in the context of the modified laminar flamelet PDF, which is then extended to the LEM formulation. The relevant parameters governing the behavior of the distributions are the mean $(\bar{c})$ and variance $\left(\bar{c}^{2}\right)$, as previously mentioned by Eq. 5 . Truncations of the profiles in the spatial domain are applied to the one-dimensional flame profiles, leading to changes in both $\bar{c}$ and $\bar{c}^{\prime 2}$. The mean of the distribution increases for every point removed from the unburnt mixture $(c=0)$ boundary, whist the opposite is true for points removed from the burnt mixture $(c=1)$ boundary. Moreover, the variance decreases for every point removed from either boundary.

Figure 4(a) demonstrates an example of these truncations. The symbols correspond to the truncation limits, $\left(x_{1}, c_{1}\right)$ and $\left(x_{2}, c_{2}\right)$, where only the cells within the interval are retained. Arbitrary values of mean and variance are set to $\bar{c}=0.50$ and ${\overline{c^{\prime}}}_{n}^{2}=0.39$ for illustrative purposes. The modified laminar flamelet PDFs with the prescribed means and variances can then be constructed with the truncated flame profiles according to methods described by previous work [1]. An example of the modifield laminar flamelet PDF of $\bar{c}=0.50$ and ${\overline{c^{\prime}}}_{n}^{2}=0.39$ is shown in Figure 4(b). Crucially, it can be deduced that there exists one unique modified laminar flamelet PDF for every mean and variance combination.

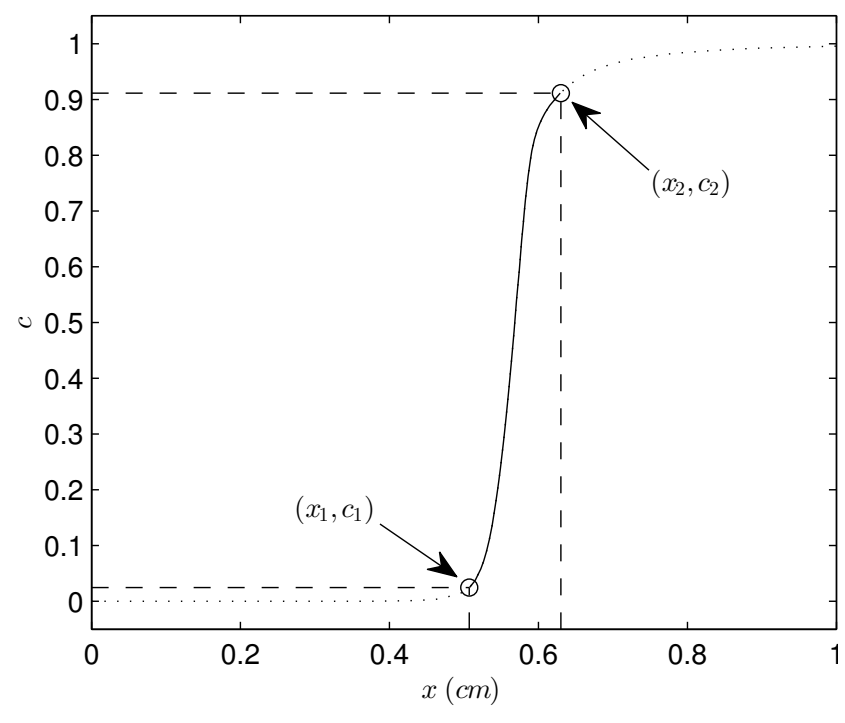

(a) Truncated laminar flame profile

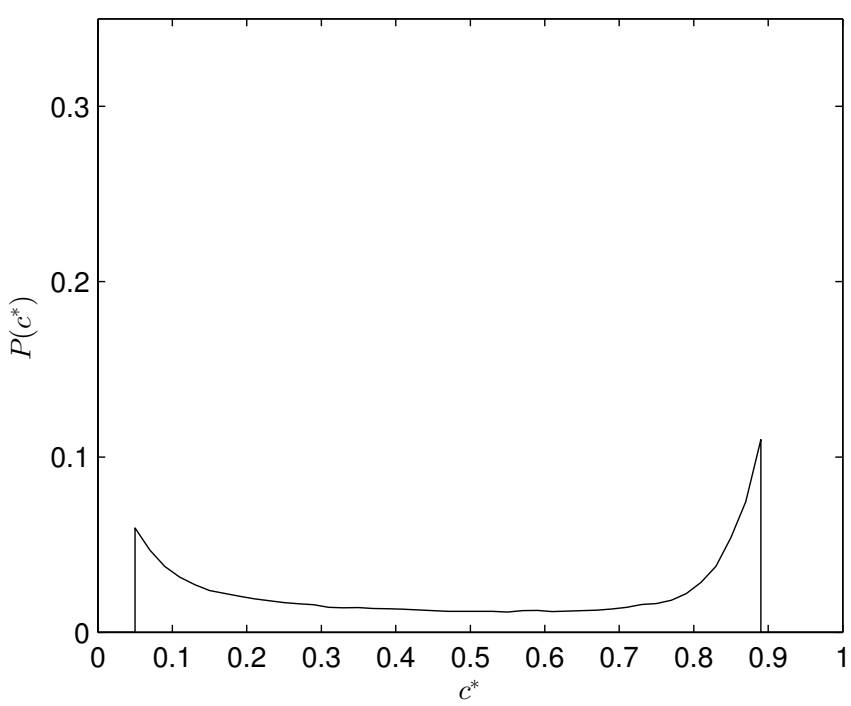

(b) Modified laminar flamelet PDF

Figure 4. (a) $\left(x_{1}, c_{1}\right)$ and $\left(x_{2}, c_{2}\right)$ mark the truncation limits; only the cells within the interval are retained. (b) The modified laminar flamelet PDF with $\bar{c}=0.50$ and ${\overline{c^{\prime}}}_{n}^{2}=0.39$ constructed using the truncated flame profile is shown.

The LEM PDFs are constructed in a similar manner compared to the modified laminar flamelet PDFs. Instead of one unique, steady, laminar temperature profile, the LEM generates transient temperature profiles; however, each LEM temperature profile is equally valid and must be equally weighted in an ensemble average to arrive at the final PDF model. Consequently, the truncation strategy prescribed for the modified laminar flamelet profiles to obtain the desired mean and variance is applied to each LEM temperature profile. Here, when a $\left(x_{1}, x_{2}\right)$ pair is fixed in real space, averaging together the many LEM realizations leads to a particular mean and variance of progress variable. In principle then, one could vary the positions of $x_{1}$ and $x_{2}$ for each temperature profile until the desired mean and variance for the PDF is achieved. In practice, all possible discrete $\left(x_{1}, x_{2}\right)$ combinations are used to populate our PDF lookup table, which is stored as a function of the mean and variance. During subsequent RANS and LES 
calculations, this table is called and interpolated as needed to obtain the PDF for any required mean and variance combination.

Figure 5(a) illustrates two truncated LEM profiles that correspond to PDFs with $\bar{c}=0.5$ and ${\overline{c^{\prime}}}_{n}^{2}=$ 0.33, similar to the laminar flame shown in Figure 4(a). The truncation positions are shifted for individual

temperature profiles because the flame changes with time. The final PDF model is acquired by averaging a number of PDFs built from such truncated LEM flames. Figure 5(b) illustrates an example of the converged solution constructed by averaging 10,000 LEM PDFs at a relatively low turbulent Reynolds number. It is apparent the LEM PDF displays peaks of lower magnitude at each boundary than the modified laminar flamelet PDF model. This smoothing effect is a direct consequence of the application of triplet maps to the temperature profiles. The mapping introduces variations in the temperature gradients at a given temperature (Figure 3(b)); as a result, the probability of the flame existing at any given state varies with different temperature profiles. When averaged, the PDF decreases to zero at each boundary more gradually [11].

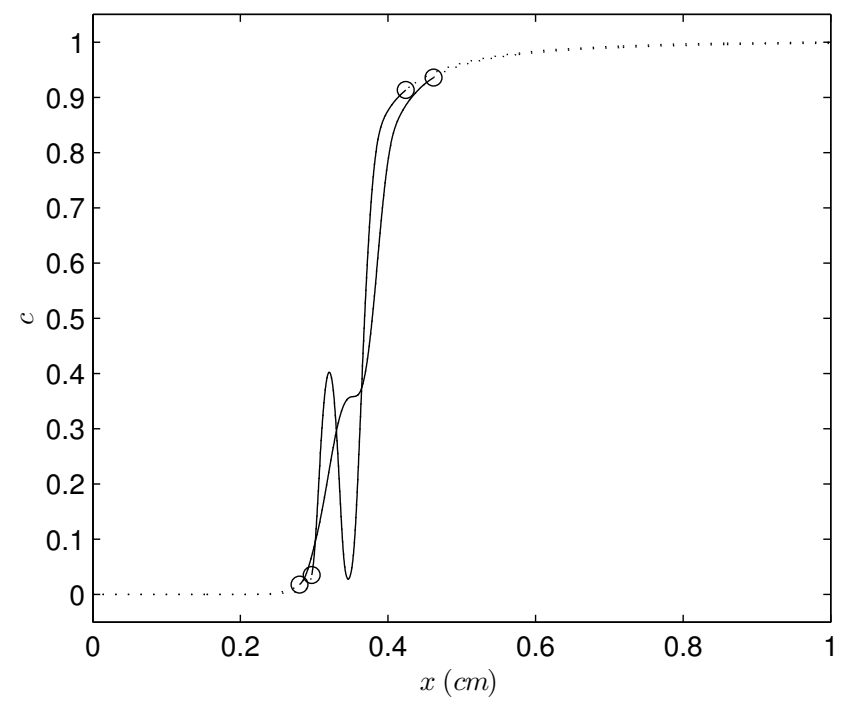

(a) Truncated instantaneous LEM flame profiles

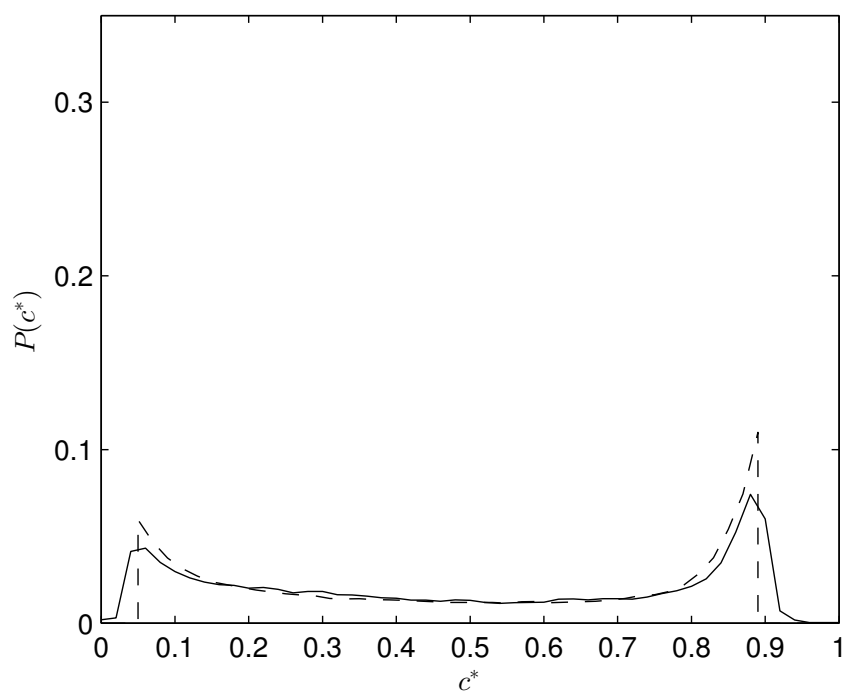

(b) Modified laminar flamelet and LEM PDFs

Figure 5. (a) The portions to be retained are within the intervals delimited by the circles. The four truncation positions are selected such that the resultant PDF would have $\bar{c}=0.5$ and ${\overline{c^{\prime}}}_{n}^{2}=0.39$. The truncation boundaries are different for each temperature profile because of the transient effects. (b) The modified laminar flamelet (dash) and LEM (solid) PDFs of similar mean and variance are shown.

\section{Results}

\subsection{Probability Density Function}

The LEM PDF models spanning a range of distribution means and variances are illustrated by Figure 6 . This study reveals that the characteristics of the PDF models for all ten prototype flames display remarkable similarities across the scope of test scenarios despite a three-order change of magnitude in turbulent fluctuation intensity and length scale on the Borghi diagram. The PDFs tend to become slightly less bivariate as the turbulence intensities increase. This effect is particularly noticeable for cases with means and normalised variances having values close to 0.5 .

The PDF distributions captured at various axial positions for the three experimental flames (SwB1-3) operating at different swirling conditions are illustrated by Figures 7-9. The LEM PDFs tabulated as a function of $c^{*}$ from one particular prototype flame has been superimposed for direct comparison between the model and the swirl burner data ${ }^{2}$. In general, a high degree of similarity can be observed between the experimental and LEM PDFs in overall features, magnitudes and peak positions. The $3 \times 5$ panels

\footnotetext{
${ }^{2}$ LEM prototype flame $1 \mathrm{~b}$ is selected for this demonstration. However, it is evident from Figure 6 that the location on the Borghi diagram does not significantly influence the resulting LEM PDF for a given variance and mean combination.
} 
correspond to different distances from the stabilisation point of the flame and radial positions across the flame brush, which are characterised by various combinations of $\bar{c}$ and $\bar{c}_{n}^{2}$.

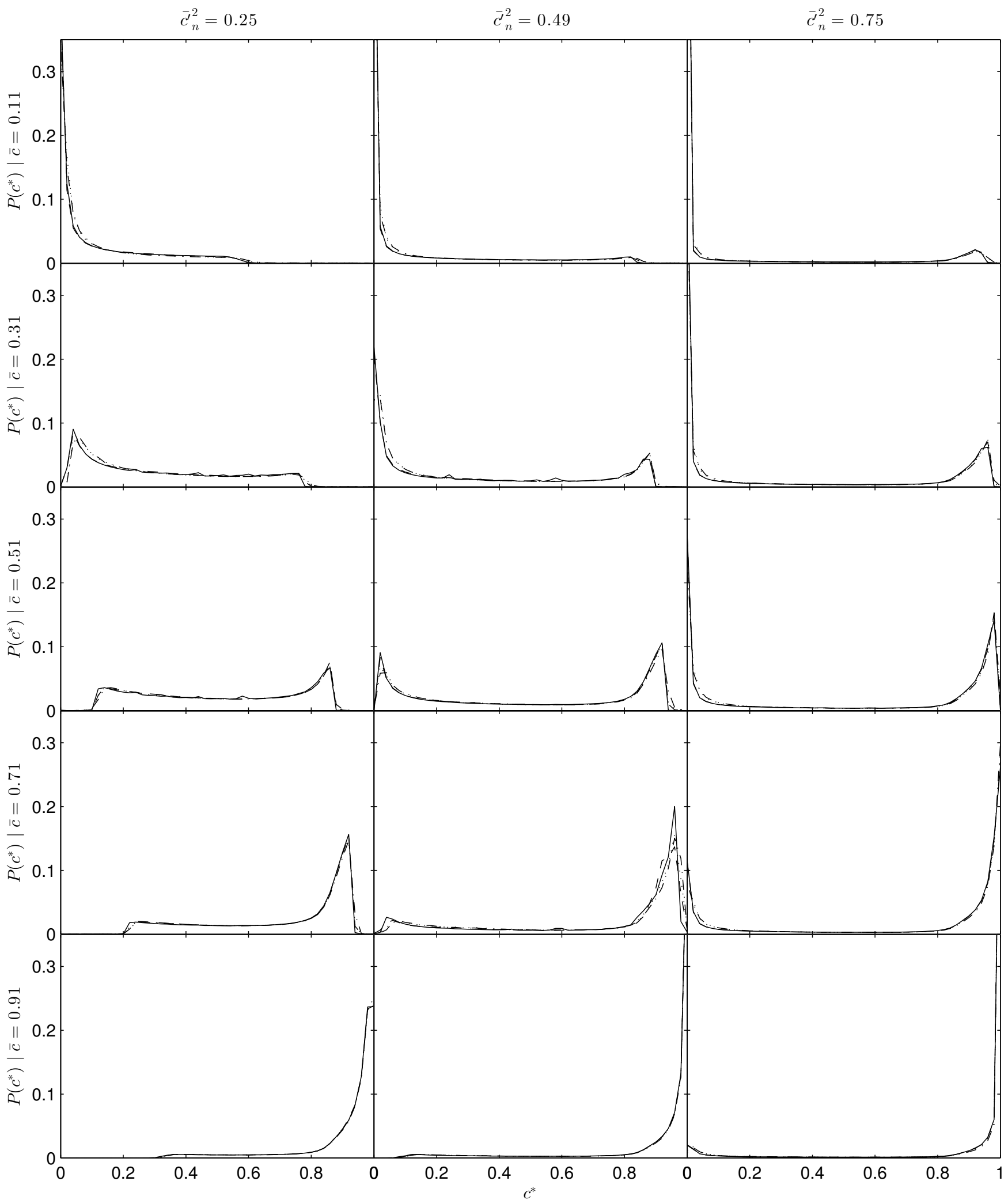

Figure 6. PDF models at various $\bar{c}$ and $\bar{c}_{n}^{\prime 2}$ are shown; each row represents one value of $\bar{c}$ and three values of $\overline{\bar{c}^{\prime}}{ }_{n}^{2}$ (ranging from left to right: $0.25,0.49$ and 0.75 ). Vertical and horizontal axis on each graph represent the probability and the progress variable, respectively. Solid: case $4 b$, dash: case $3 b$, dash dot: case $2 b$, dot: case $1 b$ (notation is in accordance with Figure 2).

Starting with SwB1 (Figure 7), the model PDFs are well matched for all normalized variance values below a distribution mean of approximately 0.7. At low $\bar{c}$ (left columns), we are at the reactant edge 
of the flame brush, and the PDF peaks at $c^{*}=0$. This agrees with the calculated LEM PDFs, but the measured PDFs are somewhat wider than the calculations suggest. At the intermediate values of $\bar{c}$, we find two low peaks, one at zero and one around 0.8-0.9, which are well captured by the LEM model, even at low variance levels. Closer to the product end of the flame brush (right columns), the PDF peak appears not at unity, but at slightly lower values, between 0.8 and 0.9 , much like the experimental values. The peak at lower values than full reaction progress is attributed to the limited time for completion of reaction due to turbulent mixing of cold and hot gases. However, heat transfer to the base of the flame can also contribute to the lowering of the extent of reaction.

Proceeding to the first swirl flame (Figure 8), it can be seen that the LEM and experimental PDFs share as many similarities as with the no swirl case. The model and experiment agree fairly well for most values of variances at a distribution mean of less than approximately 0.7. The experimental PDFs tend to be slightly less bivariate in comparison to the no swirl case, reflected by the decreasing distribution variance. An interesting difference can be observed for the results having $\bar{c} \approx 0.7$ and $\bar{c}_{n}^{\prime 2}<0.4$. The probability of $c^{*}$ continues to be non-zero towards the 0 boundary for the experimental PDFs whereas the LEM PDFs tend to go to zero. This could suggest that the swirling flow may prolong the time during which the flame spends in the preheat layer, effectively increasing the probability of finding the flame at a low $c^{*}$ state and decreasing the gradient of the progress variable around this region of the flame.

For the SwB3 flame (Figure 9), the LEM and experimental PDFs are typically well matched for distributions with $0.3<\bar{c}<0.7$. For PDFs with low values of $\bar{c}(\bar{c} \approx 0.11)$, it can be seen that the experimental PDF peaks have lower magnitudes and in some cases, are shifted towards a non-zero $c^{*}$ value $\left(c^{*}=0.05\right)$. More so, the distributions appear to be slightly wider than the LEM counterparts. This suggests that increasing the swirl number of the flame may have greater impact on the colder regions of the flame, where the conditional mean is low - such a behavior is not apparent in the SwB1 and SwB2 cases. Overall, the PDFs for all cases, with and without swirl, are still captured for the corresponding combination of means and variances. However, the agreement is generally better for the intermediate values of $\bar{c}$ than for the extremes.

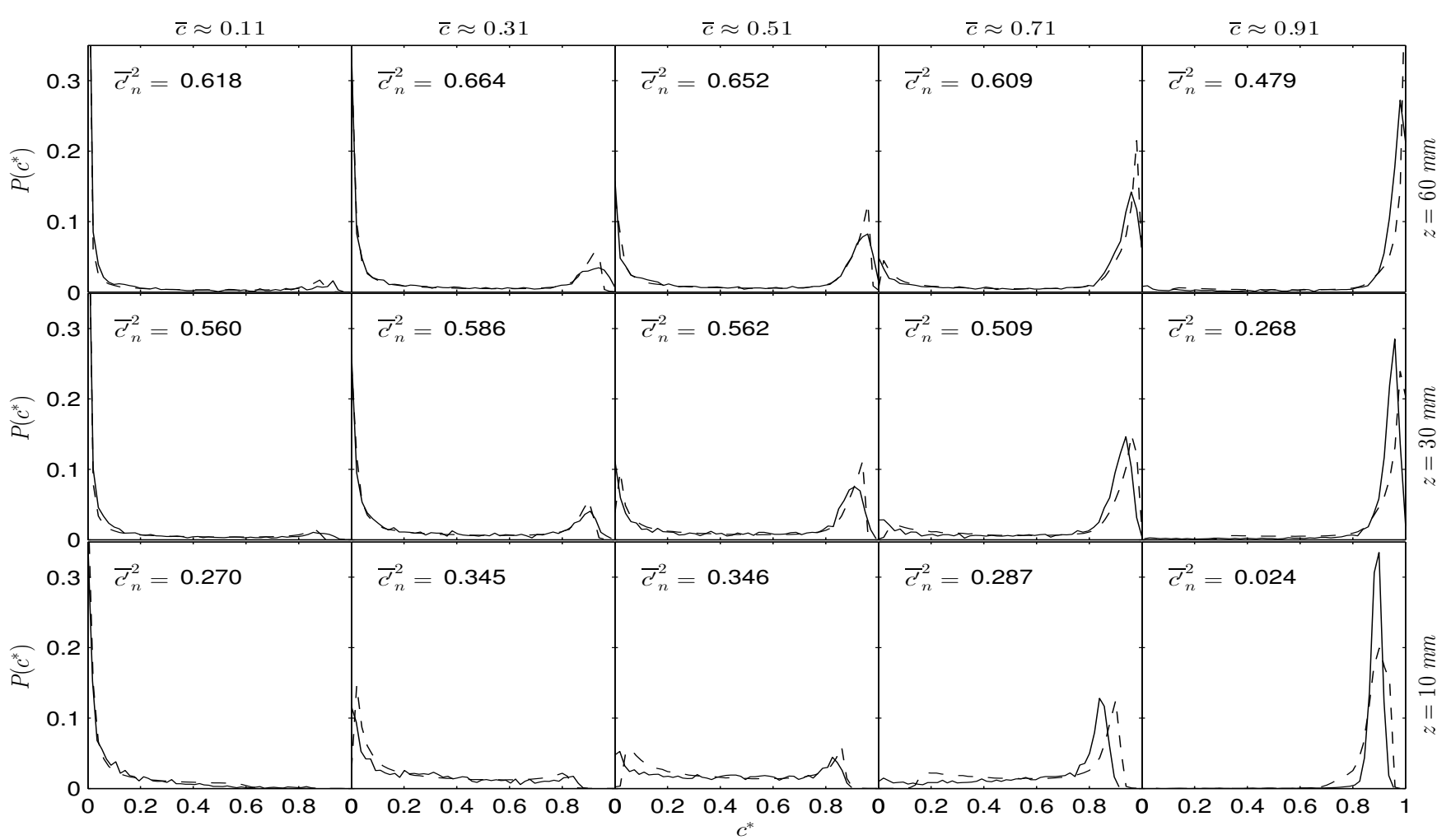

Figure 7. PDFs measured from the SwB1 flame at various axial locations conditioned by different distribution means, $\bar{c}$ (solid). The corresponding LEM PDFs of similar $\bar{c}$ and $\bar{c}_{n}^{\prime 2}$ are superimposed (dash). 


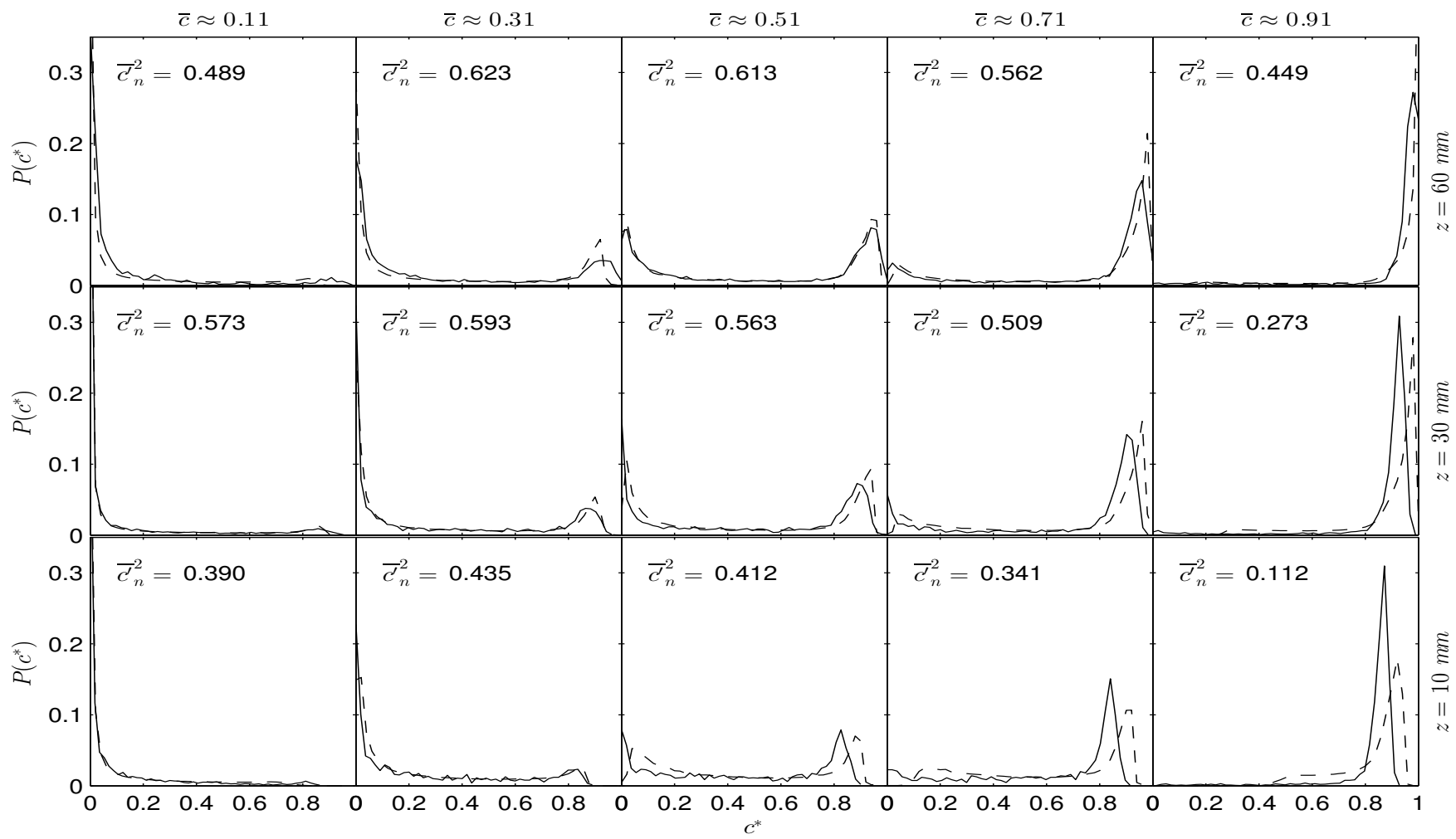

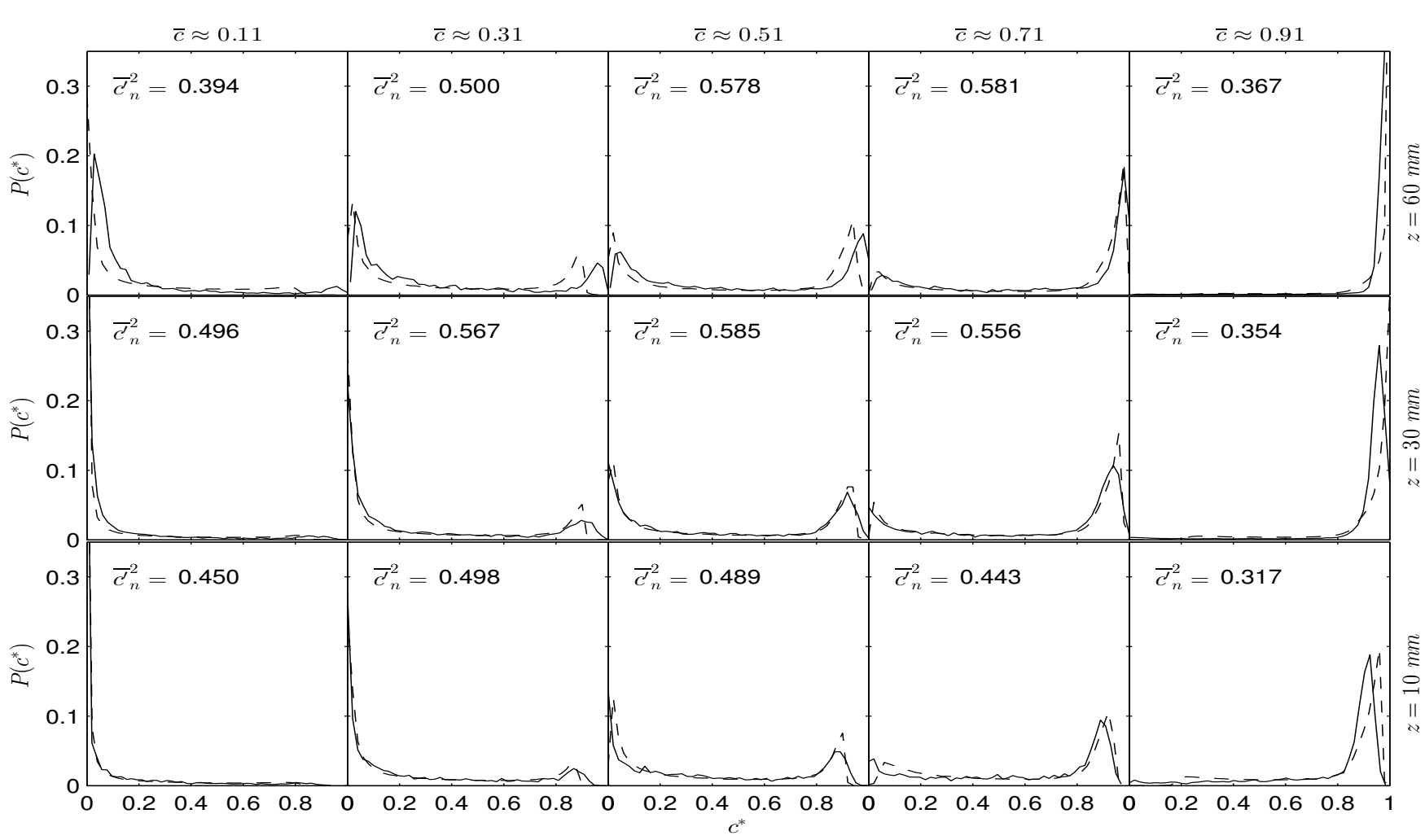

Figure 8. PDFs measured from the SwB2 flame at various axial locations conditioned by different distribution means, $\bar{c}$ (solid). The corresponding LEM PDFs of similar $\bar{c}$ and $\bar{c}_{n}^{\prime 2}$ are superimposed (dash).

Figure 9. PDFs measured from the SwB3 flame at various axial locations, conditioned by different distribution means, $\bar{c}$ (solid). The corresponding LEM PDFs of similar $\bar{c}$ and ${\overline{c^{\prime}}}_{n}^{2}$ are superimposed (dash). 


\subsection{Scalar Dissipation Rate}

A relationship can be established between the conditional scalar dissipation rate $\left(\overline{\chi_{c} \mid c^{*}}\right)$ and the PDF of the reaction progress variable $\left(P\left(c^{*}\right)\right)[14]$,

$$
\bar{\chi}_{c}=\int_{0}^{1}\left(\overline{\chi_{c} \mid c^{*}}\right) P\left(c^{*}\right) d c^{*},
$$

where $\bar{\chi}_{c}$ is the unconditional mean scalar dissipation rate, or simply the mean scalar dissipation rate. Equation 7 is particularly interesting to our analysis because the LEM temperature profiles can be used to construct both $\overline{\chi_{c} \mid c^{*}}$ and $P\left(c^{*}\right)$. A detailed description of the methodology used in constructing the PDF and SDR models can be found in [11].

There are two observable behaviors from the conditionally averaged SDR models from the LEM. First, as the characteristic flame properties are shifted upwards in the Borghi diagram (increasing $u^{\prime} / S_{L}$ ), the peak magnitudes of the dissipation rates tend to decrease. Second, if the characteristic flame properties are shifted rightward in the Borghi diagram (increasing $l_{0} / \delta_{f}$ ), the peak magnitudes of the SDRs tend to increase towards the limit of a laminar flame. It is difficult to determine from the current simulation results whether the flame broadening (increasing $u^{\prime} / S_{L}$ ) or the integral scale increment (increasing $l_{0} / \delta_{f}$ ) effect is dominant within a specific combustion regime. It is clear, however, that the processes will compete with one another in general, leading to relatively unchanged conditionally averaged SDR distributions for the special case of isotropic, homogeneous flames.

For a given chemical mechanism and set of LEM parameters, there should be one pseudo-invariant conditional scalar dissipation rate (cSDR) averaged from the temperature profile datasets. This cSDR can then be convolved with the companion PDF to arrive at the unconditional mean SDR via Equation 7. To reduce simulation time during practical implementation for complex combustion models, the values of the unconditional mean SDR can be pre-tabulated at desired combinations of means and variances of the reaction progress variable for efficient retrievals. The same LEM datasets from the PDF study can be used to generate one conditionally averaged SDR model for each of the ten prototype flames. The results are presented in Figure 10.
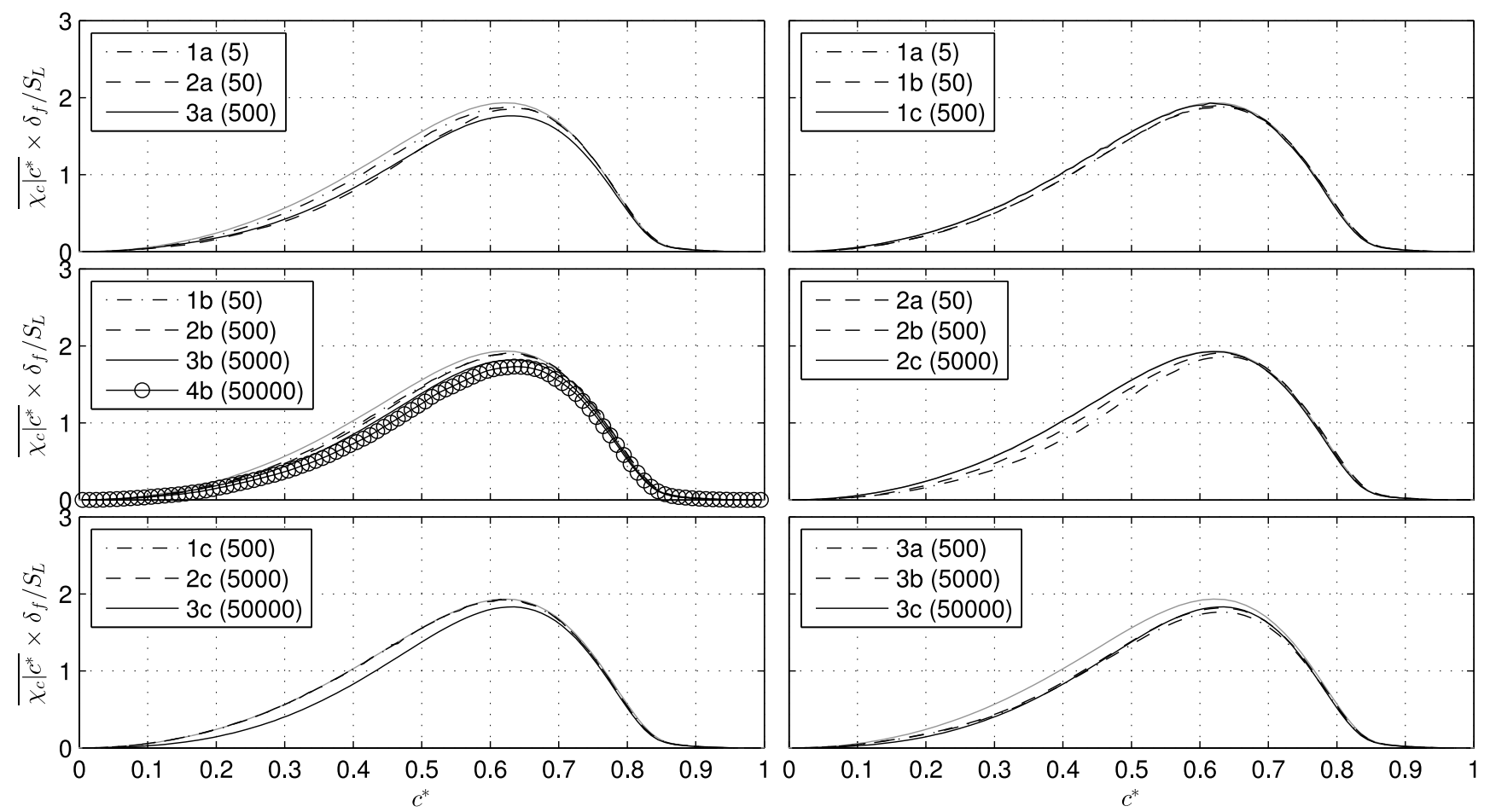

Figure 10. Non-dimensionalized conditional average of the SDR models from the ten prototype flames. The left and right columns illustrate changes in the turbulent fluctuations and integral length scales, respectively. The turbulent Reynolds number of each case is recorded in the parentheses. The laminar case is displayed in light gray as a reference. 
Figure 11 illustrates the pseudo-invariant conditional SDR distributions from the experiments. The conditional SDR typically decreases with increasing swirl and appears to be most similar to the laminar limit at $z=30 \mathrm{~mm}$. This axial location roughly coincides with the intersection of the mixing and shear layers, promoting the development of a highly turbulent and dissipative region, after which the SDR
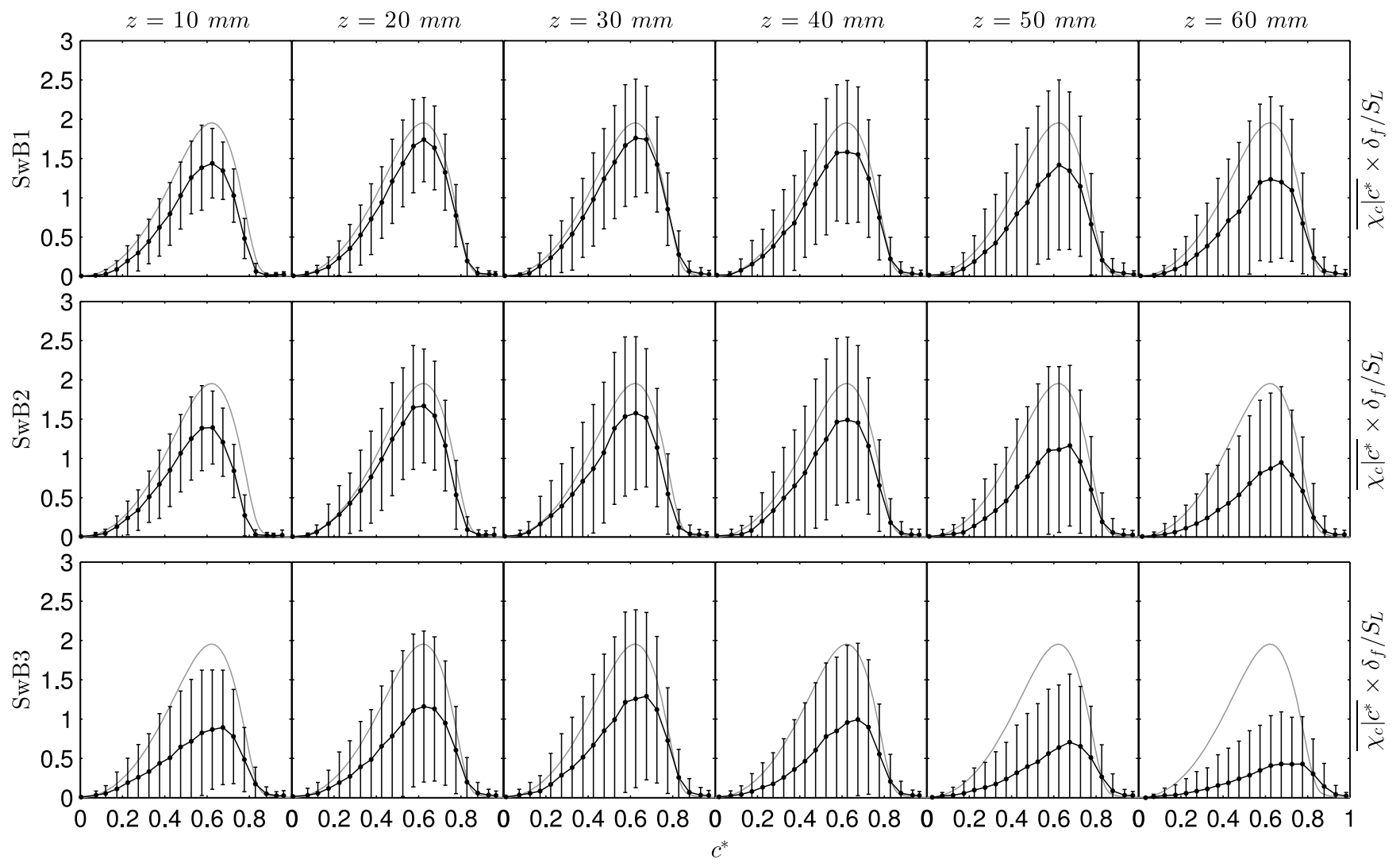
to the local turbulence characteristics, whereas the experiments indicate that there are significant changes in the SDR values; the magnitude tends to be more sensitive to the effects of swirl. Nevertheless, the shape of the distributions are well captured, with the maximum in the vicinity of $c^{*}=0.65$ to 0.75 . The maximum generally moves rightward, towards $c^{*}=0.75$, as the SDR magnitude decreases.

Figure 11. Non-dimensionalized conditional average of the SDR from the three experimental flames. Each column represents a unique axial position from the flame stabilisation point while each row represents one swirling condition. The error bars indicate $+/$ - one standard deviation from the mean. The laminar case is displayed in light gray as a reference.

As mentioned in the Introduction, several models for turbulent combustion relate the local rate of micromixing to the conditional scalar dissipation. A common approach to modelling scalar dissipation [3638 ] is to extract the functional dependence on the conditioning variable and model the conditional scalar dissipation as,

$$
\overline{\chi \mid c *}=\chi_{0} \times f\left(c^{*}\right),
$$

where $\chi_{0}$ is then calculated by some other means and is not a function of the conditioning variable. As usual, the integration of $f\left(c^{*}\right)$ over the progress variable space results in the value of unity. Here, we apply the same decomposition to our results to see if the underlying functional dependence of scalar dissipation on the conditioning variable changes - that is, to test whether or not such a decomposition might make sense.

Figure 12 depicts the functional dependence of scalar dissipation $\left(f\left(c^{*}\right)\right)$ at three axial positions from the flame stabilisation point. This figure confirms the observation that the cSDRs tend to shift rightward with increasing swirl intensity, particularly at the beginning and end of the flame brush, corresponding to the axial positions of $10 \mathrm{~mm}$ and $60 \mathrm{~mm}$. The reason for such changes in the shape of the normalised cSDR is not immediately clear from the current results; rather than speculate on the possible causes, we quickly diminish. The comparison with the LEM model is striking: in the model, there is little sensitivity 
leave this question open for future work. In general, the LEM results tend to remain close to that of the laminar flame as the model is unable to emulate the effects of swirl. Furthermore, Figure 13 illustrates the values of the normalization constant, $\chi_{0}$, in relation to the axial position for the experimental flames. It is interesting that the maximum values of $\chi_{0}$ for all of the experimental flames occur at the intersection of the mixing and shear layers near $z=30 \mathrm{~mm}$.

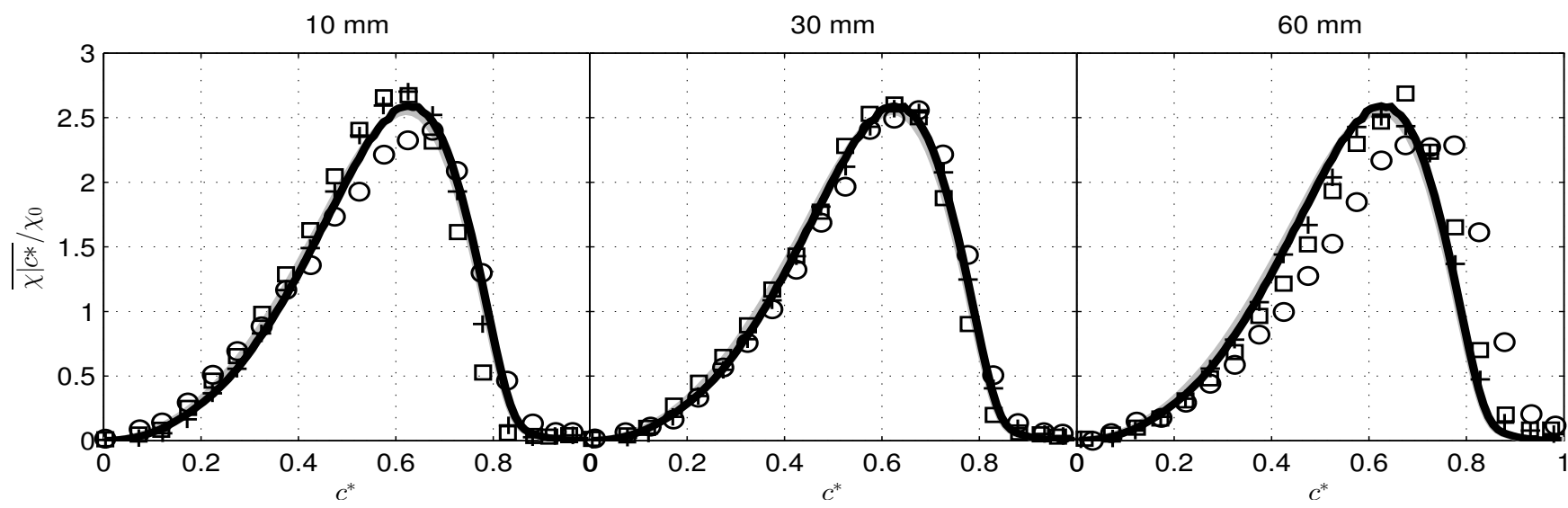

Figure 12. Functional dependence of the scalar dissipation $\left(f\left(c^{*}\right)\right)$ at three axial positions downstream from the flame stabilisation point $(10,30$ and $60 \mathrm{~mm})$ with the LEM results superimposed. The experimental flames are represented by symbols: SwB1 ('+'), SwB2 (' $\square$ '), and SwB3 ('o'). The LEM result is represented by the solid line. The laminar case is displayed in light gray as a reference.

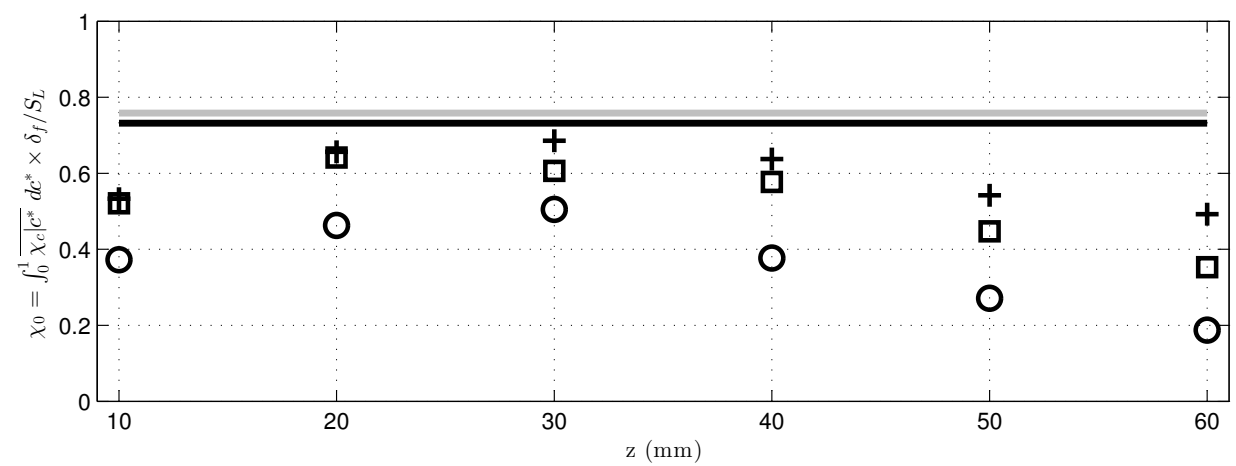

Figure 13. Values of $\chi_{0}$ in relation to the distance downstream of the flame stabilisation point. The experimental flames are represented by symbols: SwB1 ('+'), SwB2 (' $\square$ '), and SwB3 ('o'). The LEM (black line) and laminar flame (gray line) results are not dependent on the axial position.

The convolution of the pseudo-invariant conditional SDR with the appropriate PDF distribution via Equation 7 provides the solution for the unconditional mean SDR, $\bar{\chi}_{c}$, or simply the mean SDR. The modelling of this term remains as one of the final challenges in applying some combustion models to premixed flame calculations [13]. Figure 14(a) illustrates the behavior of the mean SDR as a function of the mean and variance of the progress variable as calcuated by LEM. The mean SDR peaks around $\bar{c}$ $=0.5$ and $\bar{c}_{n}^{\prime 2} \approx 0$ as the PDF and cSDR distributions have coinciding maxima for these values of $\bar{c}$ and $\bar{c}_{n}^{\prime 2}$. The experimental values can be seen in Figure 14(b), where the magnitudes of the mean SDR are evidently lower than the predicted values. The LEM predictions are best when the swirl intensity of the burner is at its lowest. This suggests that swirling flows induce a decrease in the magnitude of the SDR, both conditional and unconditional, which cannot be replicated by a one dimensional turbulence model. Moreover, recalling the PDF results from the previous section, one can conclude that swirl plays a more prominent role in the determination of the SDR than the PDF distributions. 


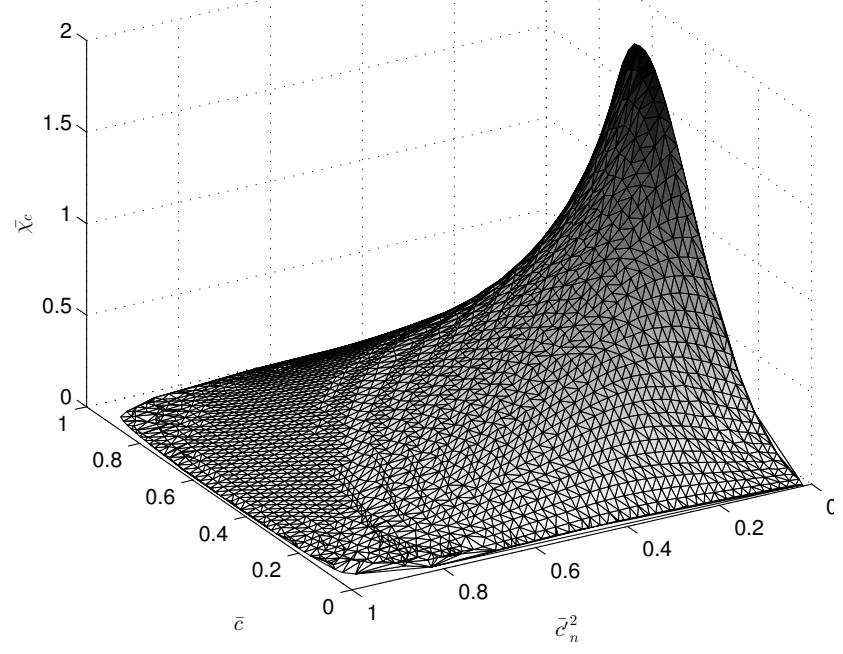

(a) LEM

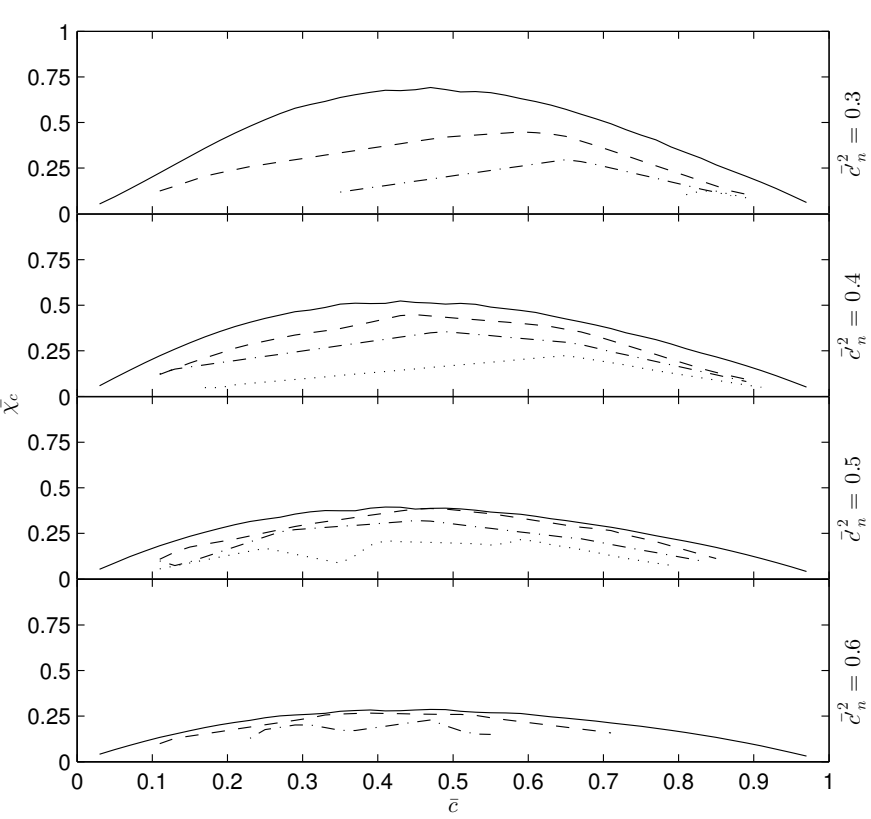

(b) Experiments and LEM

Figure 14. Non-dimensionalized unconditional mean scalar dissipation rates $\left(\bar{\chi}_{c} \times \delta_{f} / S_{L}\right)$ as predicted by (a) LEM and (b) experiments with LEM solutions superimposed at various combinations of $\bar{c}$ and ${\overline{c^{\prime}}}_{n}^{2}$. Solid: LEM; dash: SwB1; dash-dot: SwB2; dot: SwB3.

\section{Discussion}

\subsection{Probability Density Function}

The LEM formulated PDF models display minimal variations in distribution shape and magnitude with changing turbulent fluctuations and integral lengths. Typically, the PDF distributions become slightly less bivariate with increasing turbulent fluctuations. Towards the reactant boundary $\left(c^{*}=0\right)$, the turbulent fluctuations appear to increase the diffusive mixing in the preheat layer, leading to slightly faster flame development in this region. In turn, this causes a decrease in the duration of the flame residing at low values of $c^{*}$. Towards the products boundary $\left(c^{*}=1\right)$, the model suggests that the eddy interactions impede the flame from fully oxidizing towards the end of the reaction. This does not imply a reduction in the flame speed, but rather a barrier to reaching full chemical equilibrium. This effect can be observed in Figure 6, where the PDF models constructed from flames with higher turbulent fluctuations have the rightward boundaries shifted slightly towards the left. The combination of these phenomena causes the LEM PDF to deviate from the bivariate distribution seen in laminar PDF models as turbulence intensity increases, in agreement with the experimental results. The model of course neglects any potential heat transfer issues, which are present in the experiments, and may influence the approach to equilibrium at the base of the flame.

The swirl burner results (Figures 7-9) reveal that the shape of the PDFs is dependent on the distance from the base of the flame and secondarily on the amount of swirl in the flame. The dependence on distance from the base arises from the different turbulence characteristics across the flame brush as the flow evolves downstream. The variance increases with distance from the base, and with swirl, and this directly affects the corresponding PDFs. The experimental PDFs are perhaps surprisingly well captured by the two input parameters of $\bar{c}$ and ${\overline{c^{\prime}}}_{n}^{2}$. Nevertheless, once the variance and mean are determined, there are only so many sensible ways for the PDF to be arranged within the LEM context, so perhaps this justifies the good agreement.

For future implementations of the LEM PDF, where a pre-processing operation is used to populate a PDF lookup table, the data suggests that variations in the turbulent Reynolds number and integral lengths induce a rather modest effect on the shape of the PDF. It appears that it may not be necessary to add an additional dimension related to the turbulence intensity to the lookup table. Indeed, while it would be ideal to construct a table using representative conditions, using a model generated from a flame with 
lower turbulence intensities (which is the less computationally intensive option) may suffice. The number of temperature profiles required to construct converged LEM PDF models can be markedly reduced while the additional error contribution remains small. This is especially true for premixed flames with turbulent Reynolds numbers and integral lengths within an order of magnitude of the targeted flame on the Borghi

diagram. Further discretion may be required when applying different PDF models for flames exposed to more intense swirling conditions. The experimental results reveal that the overall flame gradients and hence the flame surface density function reduce in magnitude under swirl. However, such changes may not greatly alter the shape of the PDF of the progress variable because of the normalization procedure required in the construction of the LEM PDF models.

\subsection{Scalar Dissipation Rate}

As previously mentioned, there are two observable behaviors from the conditionally averaged SDRs computed by the LEM. First, as the intensity of turbulence increases, the peak magnitudes of the mean dissipation rates tend to decrease. Such an effect has been previously observed in DNS studies and is attributed to flame broadening caused by the decrease in peak temperature gradients [39]. This effect is emulated by the LEM via the interactions between the turbulent eddies and the scalar fields. These small eddies penetrate into the reaction zone, increasing the local gradients of the scalar fields. This is followed by the dissipation of the sharp discontinuities introduced in the slope of the scalar fields due to the coupled diffusion mechanism. Effectively, the flame is broadened, increasing its thickness and decreasing the gradients. Second, as the turbulent length scale increases at constant turbulent fluctuation intensity, the peak magnitudes of the SDRs tend in increase towards the limit of a laminar flame ${ }^{3}$. Such an effect could be connected to the increasing Kolmogorov length associated with the integral scale increment. An increase in the Kolmogorov length means the smallest eddies will become too large to effectively change the local structure of the flame. In another words, the eddies have more difficulty penetrating the preheat layer and the reaction zone, thus reducing the interaction between the vortices and the flame. With a smaller number of interactions, the pseudo-turbulent SDR distributions will become more similar to the laminar SDR distribution.

It is important to emphasise that the current numerical results do not include the effect of strain. Additional modelling parameters would need to be introduced to the transport equations for such calculations. Previous studies have shown that regions under highly positive strain are correlated with regions of increased scalar dissipation, though this is not an exclusive association [40, 41]. For turbulent flames exposed to high strain rates, neglecting this effect may lead to non-negligible changes to both the PDF and SDR distributions.

The pseudo-invariant conditional SDR distributions and mean SDR values from the experimental flames show a more pronouced decrease in magnitude with increasing swirl effects. It appears that the intersection of the mixing and shear layers near $z=30 \mathrm{~mm}$ leads to quickly diminishing gradients of $c$ for positions downstream of this location. This suggests that additional parameters which quantify the dissipative effects of mixing and swirling may be necessary in order to accurately model the conditional SDR distributions. Moreover, the entrainment of cold air into the premixed flame caused by the increase in fluid motion may induce changes in the temperature based reaction progress variable that the LEM cannot currently simulate. It is, however, curious that the PDF distributions from both the LEM and experiments reveal far less susceptibility to changes when exposed to physical effects, such as entrainment of air and swirl, in comparison to the conditional SDR distributions.

Nevertheless, the quantity that requires closure in combustion models is the unconditional mean SDR, defined by Equation 7. In this regard, it appears that the LEM can reproduce values of $\bar{\chi}_{c}$ well within $50 \%$ of the experimental equivalent, but the accuracy depends primarily on the swirl number of the flow. For most points, the LEM over-estimates the value of $\bar{\chi}_{c}$, which is understandable considering the conditional SDR distributions from the model indicate higher magnitudes compared to the experiments. Further research could explore the possibility of including additional modelling parameters into the LEM formulated conditional SDR model according to physical effects governing the flow field. In this case,

\footnotetext{
${ }^{3}$ For unstrained flows, the maximum conditionally averaged SDR distribution corresponds to the laminar flame with the same chemistry; any turbulence interactions decrease the magnitude of the SDR distribution with respect to the laminar limit.
} 
perhaps a post-processing correction can account for the entrainment of air induced by the swirl. This could amount to applying a simple correction factor to the magnitude of the cSDR, which is calculated as a function of the local anisotropy.

\section{Conclusion}

This study investigates the effects of variation in swirl and turbulence intensity on the probability distribution of the reaction progress variable for a series of globally lean, turbulent premixed flames, both experimentally and numerically.

The PDF models constructed from the LEM simulations indicate that increasing turbulence intensity has a rather modest impact on the distributions. The minor changes are only observed at medium values (approximately 0.5) of means and normalized variances. The PDFs from the experimental flames show a bivariate distribution for all cases, with peaks in the unburned and burned regions. The thermal flame thickness is generally larger than the unstrained (and strained) laminar flame thickness. The data therefore suggests that the thermal gradient is smeared by turbulent diffusion at scales on the order of the flame thickness. The LEM PDF model demonstrates good agreement with the experimental results in terms of nominal changes with turbulence. Particularly, it is able to capture the overall shape and the effect of smoothing towards the left and right boundaries of the PDF distributions.

The LEM results suggest that the conditional SDR would decrease towards the upper regions of the Borghi diagram and would increase in peak magnitudes towards the unstrained laminar limit with increasing integral scales. The experimental conditional SDR for all axial locations of the flame brush shows a greater decrease in magnitude than predicted by the numerical model, though this could be partially caused by an interaction between shearing and mixing layers within a specific region of the swirl flames, leading to greater dissipation downstream of this location. The values for the unconditional mean SDR predicted by LEM are typically within $50 \%$ of the experimental values. Moreover, the accuracy of the numerical model seems to be dependent on the swirl number of the flow.

Overall, the results point towards the idea that the PDF for premixed flames remains relatively steady under a variety of turbulent conditions, including changes in the integral length, velocity fluctuation and swirl number. As a consequence, it appears to be practical to use a representative pseudo-turbulent PDF model for a range of turbulent conditions. Future studies could focus on understanding the physical interpretation behind the relative invariance in the PDF model with variations in both the turbulent fluctuations and integral length scales. Another possible direction is to determine if this invariance also extends to other fuels in the premixed combustion regime. Swirl appears to have greater influence on the local flame gradients; this, in turn, leads to more substantial changes in the SDR, which the model presented here cannot capture in its current form. Perhaps a practical solution in the near future would be to implement a correction factor to the LEM formulated SDR models based on the local anisotropy of the flow field.

\section{Acknowledgements}

The authors wish to express their gratitude to the Natural Science and Engineering Research Council of Canada for partial funding of the numerical work described herein. The measurements at Sandia National Labs were sponsored by the United States Department of Energy, Office of Basic Energy Sciences, Division of Chemical Sciences, Geosciences and Biosciences. We thank Dr. Robert Barlow of Sandia National Laboratories for allowing the use of the database acquired at his laboratory, and for useful comments on the initial draft manuscript. M. Mustafa Kamal acknowledges funding from University of Engineering and Technology Peshawar (Pakistan).

\section{References}

[1] B. Jin, R. Grout, W. Bushe, Conditional Source-Term Estimation as a method for chemical closure in premixed turbulent reacting flow, Flow, Turbulence and Combustion 81 (2008) 563-582.

[2] J. V. Oijen, L. D. Goey, Modelling of premixed laminar flames using flamelet-generated manifolds, Combustion Science and Technology 161 (1) (2000) 113-137.

[3] P. Domingo, L. Vervisch, S. Payet, R. Hauguel, DNS of a premixed turbulent V flame and LES of a ducted flame using a FSD-PDF subgrid scale closure with FPI-tabulated chemistry, Combustion and Flame 143 (4) (2005) $566-586$.

[4] O. Gicquel, N. Darabiha, D. Thévenin, Laminar premixed hydrogen/air counterflow flame simulations using flame prolongation of ILDM with differential diffusion, Proceedings of the Combustion Institute 28 (2) (2000) 1901-1908. 
[5] M. M. Salehi, W. K. Bushe, Presumed PDF modeling for RANS simulation of turbulent premixed flames, Combustion Theory and Modelling 14 (3) (2010) 381-403.

[6] M. M. Salehi, W. K. Bushe, N. Shahbazian, C. P. Groth, Modified laminar flamelet presumed probability density function for LES of premixed turbulent combustion, Proceedings of the Combustion Institute 34 (1) (2013) 1203-1211.

[7] C. Fenimore, Formation of nitric oxide in premixed hydrocarbon flames, Symposium (International) on Combustion 13 (1) (1971) 373 - 380, thirteenth symposium (International) on Combustion Thirteenth symposium (International) on Combustion.

[8] K. Bray, M. Champion, P. Libby, N. Swaminathan, Finite rate chemistry and presumed PDF models for premixed turbulent combustion, Combustion and Flame 146 (4) (2006) 665 - 673.

[9] T. M. Smith, S. Menon, One-dimensional simulations of freely propagating turbulent premixed flames, Combustion Science and Technology 128 (1-6) (1997) 99-130.

[10] V. Sankaran, S. Menon, Structure of premixed turbulent flames in the thin-reaction-zones regime, Proceedings of the Combustion Institute 28 (1) (2000) 203 - 209.

[11] H. Tsui, W. Bushe, Linear-eddy model formulated probability density function and scalar dissipation rate models for premixed combustion, Flow, Turbulence and Combustion 93 (3) (2014) 487-503.

[12] J. Duclos, D. Veynante, T. Poinsot, A comparison of flamelet models for premixed turbulent combustion, Combustion and Flame 95 (1 - 2) (1993) 101-117.

[13] D. Veynante, L. Vervisch, Turbulent combustion modeling, Progress in Energy and Combustion Science 28 (3) (2002) 193-266.

[14] S. Amzin, N. Swaminathan, J. W. Rogerson, J. H. Kent, Conditional moment closure for turbulent premixed flames, Combustion Science and Technology 184 (10-11) (2012) 1743-1767.

[15] J. Zhang, F. Gao, G. Jin, G. He, Conditionally statistical description of turbulent scalar mixing at subgrid-scales, Flow, Turbulence and Combustion 93 (1) (2014) 125-140.

[16] M. S. Sweeney, S. Hochgreb, M. J. Dunn, R. S. Barlow, The structure of turbulent stratified and premixed methane/air flames I: Non-swirling flows, Combustion and Flame 159 (9) (2012) 2896-2911.

[17] M. Sweeney, S. Hochgreb, R. Barlow, The structure of premixed and stratified low turbulence flames, Combustion and Flame 158 (5) (2011) 935-948.

[18] M. Sweeney, S. Hochgreb, M. Dunn, R. Barlow, A comparative analysis of flame surface density metrics in premixed and stratified flames, Proceedings of the Combustion Institute 33 (1) (2011) 1419-1427.

[19] M. S. Sweeney, S. Hochgreb, M. J. Dunn, R. S. Barlow, The structure of turbulent stratified and premixed methane/air flames II: Swirling flows, Combustion and Flame 159 (9) (2012) 2912-2929.

[20] R. Zhou, S. Balusamy, M. S. Sweeney, R. S. Barlow, S. Hochgreb, Flow field measurements of a series of turbulent premixed and stratified methane/air flames, Combustion and Flame 160 (10) (2013) 2017-2028.

[21] F. Proch, A. M. Kempf, Numerical analysis of the cambridge stratified flame series using artificial thickened flame LES with tabulated premixed flame chemistry, Combustion and Flame 161 (10) (2014) 2627 - 2646.

[22] V. Katta, W. M. Roquemore, C/H atom ratio in recirculation-zone-supported premixed and nonpremixed flames, Proceedings of the Combustion Institute 34 (1) (2013) 1101 - 1108.

[23] A. R. Kerstein, Linear-eddy modeling of turbulent transport. Part 2: Application to shear layer mixing, Combustion and Flame 75 (3-4) (1989) 397-413.

[24] A. R. Kerstein, Linear-eddy modeling of turbulent transport. Part 3: Mixing and differential molecular diffusion in round jets, Journal of Fluid Mechanics 216 (1990) 411-435.

[25] A. R. Kerstein, Linear-eddy modeling of turbulent transport. Part 4: Structure of diffusion flames, Combustion Science and Technology 81 (1-3) (1992) 75-96.

[26] A. R. Kerstein, Linear-eddy modeling of turbulent transport. Part 6: Microstructure of diffusive scalar mixing fields, Journal of Fluid Mechanics 231 (1991) 361-394.

[27] A. R. Kerstein, Linear-eddy modeling of turbulent transport. Part 7: Finite-rate chemistry and multi-stream mixing, Journal of Fluid Mechanics 240 (1992) 289-313.

[28] M. Oevermann, H. Schmidt, A. Kerstein, Investigation of autoignition under thermal stratification using linear eddy modeling, Combustion and Flame 155 (3) (2008) 370 - 379.

[29] W. Chang, J. Chen, Reduced mechanisms for premixed and non-premixed combustion (1999).

URL http: //firebrand.me.berkeley.edu/griredu.html

[30] G. Smith, et al., GRI-Mech 3.0, http://www.me.berkeley.edu/gri_mech/ (1997).

[31] R. J. Kee, F. M. Rupley, J. A. Miller, CHEMKIN-II: A FORTRAN chemical kinetics package for the analysis of GasPhase chemical kinetics.

[32] R. J. Kee, F. M. Rupley, J. A. Miller, The CHEMKIN Thermodynamic Data Base (1991).

[33] D. G. Goodwin, Cantera. URL http: //www . cantera.org

[34] M. S. Sweeney, S. Hochgreb, M. J. Dunn, R. S. Barlow, Multiply conditioned analyses of stratification in highly swirling methane/air flames, Combustion and Flame 160 (2) (2013) 322-334.

[35] M. M. Kamal, R. Zhou, S. Balusamy, S. Hochgreb, Favre- and Reynolds-averaged velocity measurements: Interpreting PIV and LDA measurements in combustion, Proceedings of the Combustion Institute 35 (3) (2015) 3803 - 3811.

[36] C. Montgomery, G. Kosály, J. Riley, Direct numerical solution of turbulent nonpremixed combustion with multistep hydrogen-oxygen kinetics, Combustion and Flame 109 (1-2) (1997) 113 - 144. 
[37] N. Swaminathan, R. Bilger, Assessment of combustion submodels for turbulent nonpremixed hydrocarbon flames, Combustion and Flame 116 (4) (1999) $519-545$.

[38] N. Swaminathan, R. W. Bilger, Scalar dissipation, diffusion and dilatation in turbulent H2-air premixed flames with complex chemistry, Combustion Theory and Modelling 5 (3) (2001) 429-446.

[39] R. Sankaran, E. R. Hawkes, J. H. Chen, T. Lu, C. K. Law, Structure of a spatially developing turbulent lean methane-air bunsen flame, Proceedings of the Combustion Institute 31 (1) (2007) 1291-1298.

[40] P. S. Kothnur, N. T. Clemens, Effects of unsteady strain rate on scalar dissipation structures in turbulent planar jets, Physics of Fluids 17 (12) (2005) -.

[41] M. Tsurikov, Experimental investigation of the fine scale structure in turbulent gas-phase jet flows, Ph.D. thesis, University of Texas at Austin (2002). 